\title{
THE ROLE OF MASS AND ENVIRONMENT IN MULTIPLE-STAR FORMATION: A 2MASS SURVEY OF WIDE MULTIPLICITY IN THREE YOUNG ASSOCIATIONS
}

\author{
Adam L. Kraus and Lynne A. Hillenbrand \\ Department of Astrophysics, California Institute of Technology, Pasadena, CA 91125; alk@astro.caltech.edu, lah@astro.caltech.edu \\ Received 2006 December 13; accepted 2007 February 19
}

\begin{abstract}
We present the results of a search for wide binary systems among 783 members of three nearby young associations: Taurus-Auriga, Chamaeleon I, and two subgroups of Upper Scorpius. Near-infrared (JHK) imagery from 2MASS was analyzed to search for wide $\left(1^{\prime \prime}-30^{\prime \prime} ; \sim 150-4500 \mathrm{AU}\right)$ companions to known association members, using colormagnitude cuts to reject likely background stars. We identify a total of 131 candidate binary companions with colors consistent with physical association, of which 39 have not been identified previously in the literature. Our results suggest that the wide binary frequency is a function of both mass and environment, with significantly higher frequencies among high-mass stars than lower mass stars and in the T associations than in the OB association. We discuss the implications for wide binary formation and conclude that the environmental dependence is not a direct result of stellar density or total association mass, but instead might depend on another environmental parameter like the gas temperature. The binary populations in these associations generally follow the empirical mass-maximum separation relation observed for field binaries, but we have found one candidate low-mass system (USco 160611.9-193532; $\left.M_{\text {tot }} \sim 0.4 M_{\odot}\right)$ that has a projected separation $\left(10.8^{\prime \prime} ; 1550 \mathrm{AU}\right)$ much larger than the suggested limit for its mass. Finally, we find that the binary frequency in the USco-B subgroup is significantly higher than in the USco-A subgroup and is consistent with the measured values in Taurus and ChamI. This discrepancy, the absence of high-mass stars in USco-B, and its marginally distinct kinematics suggest that it might not be directly associated with the OB associations of Sco-Cen but instead represents an older analog of the younger $\rho$ Oph or Lupus associations.
\end{abstract}

Subject headings: binaries: visual — stars: low-mass, brown dwarfs — stars: pre-main-sequence

Online material: machine-readable tables

\section{INTRODUCTION}

The frequency and properties of multiple-star systems are important diagnostics for placing constraints on star formation processes and calibrating stellar evolutionary models. This has prompted numerous attempts to characterize the properties of nearby binary systems in the field. Multiplicity surveys of solartype stars (e.g., Abt \& Levy 1976; Duquennoy \& Mayor 1991) found relatively high binary frequencies $(\gtrsim 60 \%)$ and a wide range of binary separations $\left(\lesssim 10^{4} \mathrm{AU}\right)$ and mass ratios $(1$ to $\lesssim 0.1)$. This has led to the common assumption that binary systems are the primary channel for star formation. However, multiplicity surveys of lower mass M dwarfs (Fischer \& Marcy 1992; Reid \& Gizis 1997) observed marginally lower binary frequencies $(35 \%-43 \%)$, and surveys near and below the substellar boundary (Close et al. 2003; Bouy et al. 2003; Burgasser et al. 2003; Siegler et al. 2005) found substantially lower binary frequencies $(10 \%-20 \%)$ and separations (typically $\lesssim 20 \mathrm{AU}$ ) and a strong tendency toward mass ratios near unity.

These results demonstrate that field binary properties depend on mass. Unfortunately, binary properties for field stars are reported only for broad mass ranges, so they do not place strong constraints on the functional form of this dependence. Various groups interpret the transition in binary properties as either a sharp break near the stellar/substellar boundary (Kroupa et al. 2003; Close et al. 2003) or a smooth mass dependence (Luhman 2004c). Also, field multiplicity surveys cannot constrain the mass dependence of substellar binary properties due to the degeneracy between brown dwarf masses and ages. Substellar companions in the field also tend to be old and intrinsically faint, so a limited range of binary mass ratios are accessible to observations. Finally, the field represents a composite population drawn from all star formation regions, so field surveys cannot probe the dependence of binary properties on initial conditions (the stellar density, total mass, or mean Jeans mass of the formation region). One solution to these problems is to extend multiplicity surveys to the nearest young uniform stellar populations: OB associations, T associations, and open clusters.

Multiplicity surveys have been conducted for many of the bright members of nearby open clusters and associations over the past decade using the Hubble Space Telescope (HST; Martin et al. 2000; Luhman et al. 2005), adaptive optics (Patience et al. 2002; Bouy et al. 2006a), and speckle interferometry (Kohler et al. 2000; R. White et al. 2007, in preparation). These surveys have confirmed many trends observed in the field, such as the high binary frequency and separations of solar-type stars (e.g., Ghez et al. 1993; Kohler et al. 2000) and the low frequency and separation of the lowest mass systems (Martin et al. 2003; Luhman et al. 2005; Kraus et al. 2005, 2006; Bouy et al. 2006b). However, they have also found some potentially interesting discrepancies. Surveys of different regions have revealed a mass dependence in binary frequency that is either smooth (Taurus-Auriga; R. White et al. 2007, in preparation) or potentially discontinuous near the substellar boundary (Upper Scorpius; Kohler et al. 2000; Kraus et al. 2005). Several systems with unusually wide separations or low mass ratios have also been found (e.g., Luhman 2004b; Bouy et al. 2006a).

High-resolution imaging techniques are typically resource intensive, so it is expensive to undertake large programs that can sample a wide range of mass with sufficient statistical significance to characterize these effects. However, wide binary systems in the nearest associations have angular separations large enough to 
TABLE 1

Nearby Young Associations

\begin{tabular}{ccccc}
\hline \hline Name & $\begin{array}{c}\text { Distance } \\
(\mathrm{pc})\end{array}$ & $\begin{array}{c}\text { Age } \\
(\mathrm{Myr})\end{array}$ & Type & Members \\
\hline Chamaeleon I ............ & 170 & $1-2$ & T association & 147 \\
Taurus-Auriga ............ & 145 & $1-2$ & T association & 235 \\
Upper Scorpius A ....... & 145 & 5 & OB association & 356 \\
Upper Scorpius B ....... & 145 & 5 & OB association & \\
\end{tabular}

${ }^{\text {a }}$ As we discuss in Appendix C, the nature of Upper Sco B is still uncertain.

resolve without these techniques. A program that exploits a uniform, high-quality seeing-limited survey could substantially enhance our understanding of the role of mass and environment in binary properties.

In this paper we present the results from a search for new young binary systems in the Two Micron All Sky Survey (2MASS), an all-sky imaging survey conducted in the near-infrared. In $\S 2$ we describe the selection of our survey sample, and we describe our data processing techniques in $\S 3$. We summarize the results of our search in $\S 4$. Finally, in $\S 5$ we compare these results to the standard paradigm of stellar multiplicity and discuss the implications for the processes of multiple-star formation.

\section{SAMPLE SELECTION}

In Table 1 we describe the young associations from which we have drawn our sample: Taurus-Auriga, Chamaeleon I, and the two proposed subgroups of Upper Scorpius. The sample regions have been selected to include all large stellar populations $(\gtrsim 100$ known members) that are not heavily embedded, are located at distances of $\leqslant 200 \mathrm{pc}$, and have ages $\lesssim 30 \mathrm{Myr}$. These criteria neglect small associations and moving groups that cannot contribute significant statistics (TW Hya, MBM 12, Chamaeleon II, $\eta$ and $\epsilon$ Cham, and the Lupus clouds), distant populations for which seeing-limited observations cannot probe sufficiently small separations (IC 348 and the subgroups of Orion), embedded populations like the $\rho$ Oph complex, and old populations in which the wide binary population may have been shaped by dynamical evolution (Praesepe, Pleiades, $\alpha$ Persei).

In Table 2 we list the association members that we have adopted as our primary sample in the multiplicity search. The regional membership of our sample has been confirmed via low-resolution spectroscopy to verify signatures of youth, so contamination of the primary sample should be negligible. As we discuss in $\S 4.2$, the surveys from which we draw our sample are likely to be incomplete due to selection biases; many of the new candidate companions found here would have been identified in previous surveys if they were complete and unbiased. This could potentially cause us to overestimate the wide binary frequency. Wide binaries would only be excluded from our sample if both com- ponents were absent from previous membership surveys, so they are less likely to have been omitted from our sample than single members. However, this effect would have been more prevalent among faint low-mass systems (where incompleteness is higher). We are testing for a decline in the binary frequency with mass, and any detection of this trend would be robust against this bias.

Saturation occurs for 2MASS sources brighter than $K \sim 8$, but the images can still be used for sources as bright as $K \sim 6$; we have neglected only the high-mass association members that are brighter than this limit, corresponding to spectral types earlier than G0. These bright stars typically have been studied with adaptive optics (e.g., Kouwenhoven et al. [2005] for the Sco-Cen complex), so analysis of 2MASS data would not contribute significant new results. We also omit all sources that do not have confirmed spectral types since we cannot estimate their mass. This criterion should eliminate most of the sources that are embedded in massive envelopes and surrounded by resolved nebulosity. Finally, four of our primary sample members are fainter than our detection limit for binary companions $(K=14.3)$, but we retain them in our sample in case they are binary companions to higher mass association members that have not yet been identified.

In the following subsections we briefly describe each association and summarize the construction of our search sample.

\subsection{Scorpius-Centaurus}

The Sco-Cen OB association consists of three distinct subgroups: Upper Scorpius (USco; 5 Myr and 145 pc), Upper Centaurus Lupus (UCL; $13 \mathrm{Myr}$ and $160 \mathrm{pc}$ ), and Lower Centaurus Crux (LCC; 10 Myr and 118 pc) (de Geus et al. 1989; de Zeeuw et al. 1999). Sco-Cen has been recognized for nearly a century as a moving group of early-type stars (e.g., Kapteyn 1914; Blaauw 1946; Bertiau 1958; Jones 1971). However, surveys to identify low-mass stellar members have been undertaken only in the past 15 years and have concentrated almost exclusively on USco. Initial surveys (Walter et al. 1994; Kunkel 1999) identified candidate members from surveys for X-ray emission, while subsequent surveys (Preibisch et al. 1998, 2001, 2002; Ardila et al. 2000; Martin et al. 2004; Slesnick et al. 2006a) used wide-field optical/NIR surveys to select candidate members with colors and magnitudes consistent with the assumed age and distance. Membership was confirmed with low- or intermediate-resolution spectroscopy to confirm indicators of youth such as lithium absorption, $\mathrm{H} \alpha$ emission, or low surface gravity. Proper-motion member identification is typically not possible for faint Sco-Cen members since their proper motions are not sufficiently distinct from those of background stars; the only major effort has been by Mamajek et al. (2002), who identified candidate $\mathrm{G}$ and $\mathrm{K}$ dwarf members of UCL and LCC based on proper motions and then confirmed their membership with low-resolution spectroscopy.

The sample sizes for UCL and LCC are marginal ( $\sim 50$ members each) and span a limited range of masses, and the associations'

TABLE 2

Confirmed Members of Nearby Young Associations

\begin{tabular}{|c|c|c|c|c|c|c|c|c|c|c|c|}
\hline Name & Region & $\begin{array}{c}\text { R.A. } \\
\text { (J2000.0) }\end{array}$ & $\begin{array}{c}\text { Decl. } \\
\text { (J2000.0) }\end{array}$ & $K$ & $J-K$ & $H-K$ & Spectral Type & $\begin{array}{l}\text { Mass } \\
\left(M_{\odot}\right)\end{array}$ & $\chi_{3}{ }^{\mathrm{a}}$ & $\begin{array}{c}\mu_{\alpha}, \mu_{\delta} \\
\left(\operatorname{mas~yr}^{-1}\right)\end{array}$ & References \\
\hline ScoPMS 005 .......... & USco-A & 155459.86 & -234718.2 & 7.03 & 0.54 & 0.16 & G2 & 1.66 & 26.57 & $-28,-38$ & Walter et al. (1994) \\
\hline ScoPMS $014 \ldots \ldots \ldots$. & USco-A & 155654.97 & -232947.8 & 10.29 & 0.93 & 0.30 & M3 & 0.36 & 1.67 & $-8,-28$ & Walter et al. (1994) \\
\hline
\end{tabular}

Note.-Units of right ascension are hours, minutes, and seconds, and units of declination are degrees, arcminutes, and arcseconds. Table 2 is published in its entirety in the electronic edition of the Astrophysical Journal. A portion is shown here for guidance regarding its form and content.

${ }^{a}$ The $\chi_{3}$ statistic is a measure of how well each object is fitted by a single point source; see $\S 3.2$. 
low galactic latitude $(|b|<20)$ results in substantial contamination from reddened background stars, so we have chosen to only consider Upper Sco. We select our sample from the surveys of Walter et al. (1994), Preibisch et al. (1998, 2001, 2002), Kunkel (1999), Ardila et al. (2000), Martin et al. (2004), and Slesnick et al. (2006a).

Brandner et al. (1996) noted that some of the objects in these surveys form a distinct subgroup in the southwest, near the border with UCL; they named the main population Upper Sco A and the subgroup Upper Sco B (hereafter USco-A and USco-B). A multiplicity survey by Kohler et al. (2000) subsequently found that these two populations might have distinct binary statistics, with a much wider mean separation in USco-B. As we show in Appendices A and B, the members of USco-B also appear to have distinct kinematics. These results suggest that USco-B should be treated as a distinct population. Based on the population kinematics and the previous dividing lines adopted by Brandner et al. (1996) and Kohler et al. (2000), we assign all sample members west of $16^{\mathrm{h}}$ and south of $-28^{\circ}$ to USco-B, and all remaining members to USco-A. It is quite likely that there is some overlap along this border, but the precision of the kinematic data does not allow us to unambiguously determine this or to establish the subgroup membership of individual sources.

We also note that two USco members, ScoPMS 008A and ScoPMS 008B, are located $\sim 15^{\prime \prime}$ from an early-type USco member, HD 142424 (A8 IV/V; de Zeeuw et al. 1999). It is possible that these stars are companions to HD 142424 and not independent primaries; since they fall within our identification range for binary companions in USco-A $\left(\leqslant 20^{\prime \prime}\right)$, we do not treat these sources as independent primaries. Kohler et al. (2000) found (and we verify) that ScoPMS 008A is itself a binary system with a separation of $\sim 1.6^{\prime \prime}$, which suggests that this could be at least a quadruple system.

\subsection{Taurus}

The Taurus-Auriga association (Taurus; 1-2 Myr; 145 pc; Bertout et al. 1999; White \& Ghez 2001) has been recognized for more than 60 years as the nearest northern site of low-mass star formation and is the home of the archetypical star T Tauri. The low-mass stellar population of Taurus-Auriga has been classified gradually over this time period (e.g., Joy 1945; Herbig 1952; Cohen \& Kuhi 1979); unlike Sco-Cen, Taurus is largely devoid of stars more massive than $1-2 M_{\odot}$.

A census of known Taurus members was presented in Kenyon \& Hartmann (1995) and has been supplemented by additional surveys to identify very low mass stellar and substellar members of Taurus-Auriga by Briceno et al. (1993, 1998, 2002), Strom \& Strom (1994), Martin et al. (2001), Luhman (2004c, 2006), Luhman et al. (2003a), Guieu et al. (2006), and Slesnick et al. (2006b). Members of Taurus-Auriga have also been confirmed in a follow-up survey of continuum (heavily veiled) sources by White \& Basri (2003) and a survey for Hyades members by Reid \& Hawley (1999). Finally, it was pointed out by R. White et al. (2007, in preparation) that the source FV Tau/c2 (Hartigan et al. 1994) was omitted from the compilation of Kenyon \& Hartmann (1995). We have constructed our Taurus source list from the Kenyon \& Hartmann (1995) census, plus all subsequent surveys.

\subsection{Chamaeleon I}

The Chamaeleon I complex (ChamI; 1-2 Myr; $160-170$ pc; Whittet et al. 1997; Wichmann et al. 1998; Bertout et al. 1999) is another nearby site of ongoing star formation. Like Taurus-
Auriga, it is composed primarily of low-mass stars and molecular clouds and possesses few high-mass stars. Much of its stellar population was identified by optical and near-infrared surveys during the 1970s and 1980s (e.g., Henize \& Mendoza 1973; Schwartz et al. 1977; Glass 1979; Baud et al. 1984).

Carpenter et al. (2002) and Luhman (2004b) have compiled censuses of known members and candidate members based on these and other surveys, and Luhman (2004b) confirmed the membership of many candidate members using optical and NIR spectroscopy. An objective prism survey of the entire cloud by Comerón et al. (2004) also confirmed four additional candidate members and identified seven new members. Finally, one candidate substellar member from the survey of Oasa et al. (1999) was spectroscopically confirmed as a ChamI member by Luhman et al. (2004). We have constructed our ChamI sample from the 151 confirmed ChamI members of Luhman (2004b), Luhman et al. (2004), and Comerón et al. (2004) with spectral types later than G0.

\subsection{Spectroscopically Confirmed Stellar Pairs}

Spectroscopic surveys of these stellar associations have identified many close $\left(<30^{\prime \prime}\right)$ pairs of members. Given the typical low surface density of association members on the sky, these stars could be gravitationally bound binary companions. We list these candidate binary systems in Table 3 . Many systems have projected separations lower than our survey's outer identification limits ( $\$ 4.1$ ); in these cases, we have removed the secondary star in each pair from our statistical sample. Candidate secondaries at wider separations are considered to be independent systems for statistical purposes.

\section{OBSERVATIONS AND DATA ANALYSIS}

\subsection{The Two Micron All Sky Survey}

2MASS (Skrutskie et al. 2006) observed $99.998 \%$ of the sky in the $J, H$, and $K_{s}$ bands over an interval of $4 \mathrm{yr}$. Each point on the sky was imaged six times and the co-added total integration time was $7.8 \mathrm{~s}$, yielding $10 \sigma$ detection limits of $K=14.3, H=$ 15.1 , and $J=15.8$. The saturation levels depend on the seeing and sky background for each image but are typically $J<9, H<$ 8.5 , and $K_{s}<8$. The pixel scale of the detector was $2^{\prime \prime}$ pixel $^{-1}$, but acquisition of multiple images allowed for subsampling to increase the effective resolution; the final pixel scale for each processed image is $1^{\prime \prime}$ pixel ${ }^{-1}$, which critically samples stellar point-spread functions (PSFs) given a typical resolution of $3^{\prime \prime}$ FWHM. The typical astrometric accuracy attained for the brightest unsaturated sources $(K \sim 8)$ is $\sim 100$ mas, and the photometric zero points are calibrated to $<0.02 \mathrm{mag}$.

The 2MASS Point Source Catalog (PSC; Cutri et al. 2003) and the processed survey images are available from the 2MASS Web site. ${ }^{1}$ We use PSC data to identify all wide $\left(>5^{\prime \prime}\right)$ visual companions to our sample members. However, the PSC does not always distinguish multiple point sources in close proximity $\left(\leqslant 5^{\prime \prime}\right)$, instead reporting only the brightest source. This suggests that wide neighbors to our sample members should be identified in the PSC, but most close neighbors are probably absent.

We address this incompleteness by working directly with the processed survey images to identify close $\left(\lesssim 5^{\prime \prime}\right)$ companions via PSF fitting photometry. From the 2MASS Web site, we extracted postage stamp $\left(60^{\prime \prime} \times 60^{\prime \prime}\right)$ and wide-field $\left(510^{\prime \prime} \times 1024^{\prime \prime}\right)$ images for each of the association members described in $\S 2$. The

\footnotetext{
1 Available at http://www.ipac.caltech.edu/2mass/.
} 
TABLE 3

Close Pairs of Confirmed Association Members

\begin{tabular}{|c|c|c|c|c|c|c|c|c|c|}
\hline \multirow[b]{2}{*}{ Primary NAME } & \multirow[b]{2}{*}{ Secondary Name } & \multicolumn{3}{|c|}{ PRIMARY } & \multicolumn{3}{|c|}{ SECONDARY } & \multirow{2}{*}{$\begin{array}{l}\text { Projected SeParation } \\
(\operatorname{arcsec})\end{array}$} & \multirow{2}{*}{$\begin{array}{l}\text { Position Angle } \\
\text { (deg) }\end{array}$} \\
\hline & & $J-K$ & $H-K$ & $K$ & $J-K$ & $H-K$ & $K$ & & \\
\hline 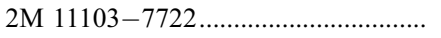 & ISO 350 & 2.00 & 0.68 & 10.03 & 2.06 & 0.7 & 10.67 & 9.51 & 145.7 \\
\hline 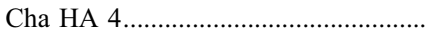 & Cha HA 10 & 1.14 & 0.41 & 11.02 & 1.06 & 0.33 & 13.24 & 20.83 & 128.5 \\
\hline 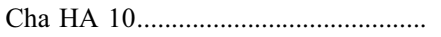 & Cha HA 11 & 1.06 & 0.33 & 13.24 & 1.04 & 0.37 & 13.55 & 19.60 & 58.9 \\
\hline 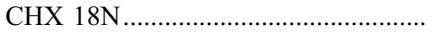 & T49 & 1.34 & 0.52 & 7.77 & 1.60 & 0.64 & 8.87 & 24.38 & 255.7 \\
\hline CHXR $14 \mathrm{~N} \ldots \ldots .$. & CHXR 14S & 0.94 & 0.23 & 9.60 & 0.98 & 0.23 & 9.75 & 28.17 & 166.6 \\
\hline 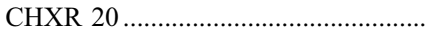 & $\mathrm{T} 22$ & 1.30 & 0.32 & 8.88 & 1.42 & 0.40 & 9.39 & 28.46 & 349.2 \\
\hline 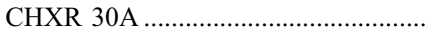 & CHXR 30B & 2.71 & 0.83 & 9.09 & 3.92 & 1.54 & 9.95 & 9.93 & 295.4 \\
\hline 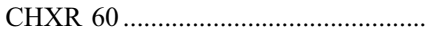 & Hn 18 & 0.99 & 0.28 & 10.58 & 1.06 & 0.31 & 10.80 & 28.28 & 220.6 \\
\hline CHXR 68A & CHXR 68B & 0.92 & 0.24 & 8.87 & 0.98 & 0.27 & 10.26 & 4.39 & 212.4 \\
\hline 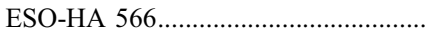 & CHSM 15991 & 1.32 & 0.42 & 11.03 & 1.91 & 0.74 & 14.14 & 23.65 & 93.9 \\
\hline 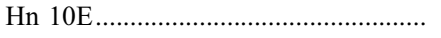 & $\mathrm{C} 1-25$ & 1.91 & 0.69 & 10.05 & 3.80 & 1.42 & 10.00 & 19.17 & 231.0 \\
\hline Hn $21 \mathrm{~W}$ & Hn $21 \mathrm{E}$ & 1.34 & 0.44 & 10.65 & 1.27 & 0.48 & 11.49 & 5.43 & 69.3 \\
\hline 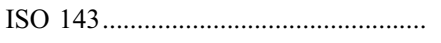 & ISO 138 & 1.48 & 0.56 & 11.10 & 1.02 & 0.43 & 13.04 & 18.16 & 223.8 \\
\hline ISO $237 \ldots$ & $\mathrm{T} 45 \mathrm{~A}$ & 2.31 & 0.82 & 8.62 & 1.33 & 0.40 & 9.24 & 28.32 & 235.7 \\
\hline $\mathrm{T} 28 \ldots \ldots \ldots \ldots$ & Cha HA 8 & 1.91 & 0.72 & 8.26 & 1.27 & 0.49 & 11.51 & 28.87 & 164.3 \\
\hline $\mathrm{T} 29 \ldots \ldots \ldots . .$. & ESO-HA 562 & 2.67 & 1.09 & 6.83 & 3.33 & 1.37 & 8.3 & 16.37 & 81.8 \\
\hline 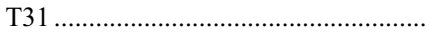 & $\mathrm{T} 30$ & 1.74 & 0.68 & 6.96 & 2.38 & 0.97 & 9.89 & 16.52 & 221 \\
\hline T34 & Cha HA 13 & 1.17 & 0.32 & 10.02 & 1.12 & 0.4 & 10.67 & 25.41 & 3.9 \\
\hline 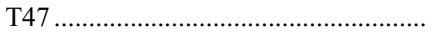 & ESO-HA 568 & 1.97 & 0.78 & 9.18 & 1.30 & 0.35 & 10.75 & 12.09 & 161.3 \\
\hline 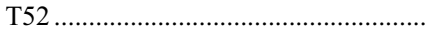 & T53 & 1.44 & 0.62 & 6.85 & 1.79 & 0.73 & 9.13 & 11.18 & 99.2 \\
\hline 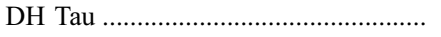 & DI Tau & 1.59 & 0.65 & 8.18 & 0.93 & 0.21 & 8.39 & 15.23 & 126 \\
\hline 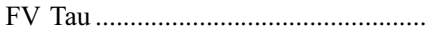 & FV Tau/c & 2.48 & 0.88 & 7.44 & 1.93 & 0.62 & 8.87 & 12.29 & 105.7 \\
\hline 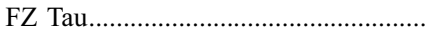 & FY Tau & 2.55 & 1.05 & 7.35 & 1.93 & 0.62 & 8.05 & 17.17 & 250.5 \\
\hline 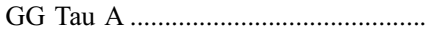 & GG Tau B & 1.31 & 0.45 & 7.36 & 1.09 & 0.42 & 9.97 & 10.38 & 185.1 \\
\hline 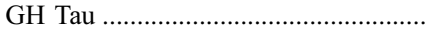 & V807 Tau & 1.32 & 0.44 & 7.79 & 1.19 & 0.40 & 6.96 & 21.77 & 15.2 \\
\hline 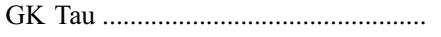 & GI Tau & 1.59 & 0.64 & 7.47 & 1.45 & 0.53 & 7.89 & 13.14 & 328.4 \\
\hline 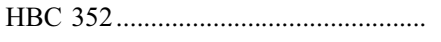 & HBC 353 & 0.51 & 0.14 & 9.58 & 0.59 & 0.14 & 9.86 & 8.97 & 70.8 \\
\hline 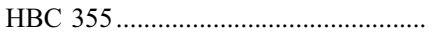 & HBC 354 & 0.62 & 0.13 & 10.20 & 0.73 & 0.15 & 11.11 & 6.31 & 298.3 \\
\hline 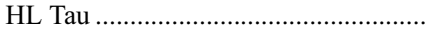 & XZ Tau & 3.21 & 1.76 & 7.41 & 2.09 & 0.86 & 7.29 & 23.31 & 91.2 \\
\hline 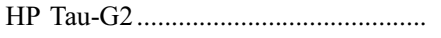 & HP Tau & 0.87 & 0.26 & 7.23 & 1.92 & 0.84 & 7.63 & 21.30 & 296.9 \\
\hline 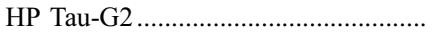 & HP Tau-G3 & 0.87 & 0.26 & 7.23 & 1.24 & 0.36 & 8.80 & 10.09 & 243.4 \\
\hline 2MASS J04554757+3028077............. & 2MASS J04554801+3028050 & 1.07 & 0.33 & 9.98 & 1.03 & 0.43 & 12.16 & 6.31 & 115.7 \\
\hline 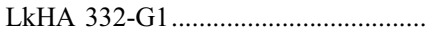 & LkHA 332-G2 & 1.64 & 0.46 & 7.95 & 1.56 & 0.44 & 8.23 & 25.88 & 254.5 \\
\hline 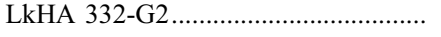 & V955 Tau & 1.64 & 0.46 & 7.95 & 1.87 & 0.66 & 7.94 & 10.51 & 35.3 \\
\hline 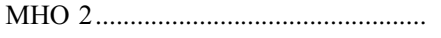 & MHO 1 & 3.73 & 1.63 & 7.79 & 4.70 & 2.10 & 7.78 & 3.93 & 333.9 \\
\hline 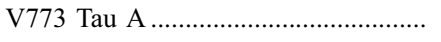 & 2MASS J04141188+2811535 & 1.28 & 0.43 & 6.21 & 1.52 & 0.69 & 11.64 & 23.38 & 215.9 \\
\hline 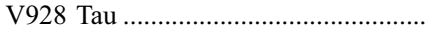 & CFHT Tau 7 & 1.43 & 0.33 & 8.11 & 1.16 & 0.41 & 10.38 & 18.25 & 228.2 \\
\hline 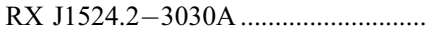 & RX J1524.2-3030B & 0.63 & 0.16 & 8.68 & 0.99 & 0.29 & 9.60 & 20.18 & 87.3 \\
\hline 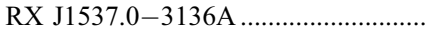 & RX J1537.0-3136B & 0.52 & 0.04 & 7.74 & 0.90 & 0.18 & 7.65 & 5.37 & 285.0 \\
\hline 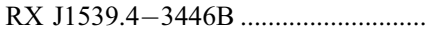 & RX J1539.4-3446C & 1.21 & 0.43 & 7.98 & 1.61 & 0.60 & 9.29 & 6.36 & 98.1 \\
\hline 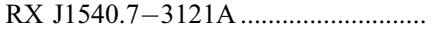 & RX J1540.7-3121B & 0.83 & 0.26 & 10.53 & 0.86 & 0.27 & 10.66 & 5.95 & 75.5 \\
\hline 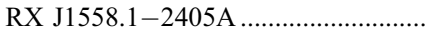 & RX J1558.1-2405B & 0.79 & 0.16 & 8.96 & 0.89 & 0.25 & 11.06 & 18.15 & 254.4 \\
\hline 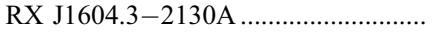 & RX J1604.3-2130B & 1.44 & 0.60 & 8.51 & 1.02 & 0.27 & 9.43 & 16.22 & 215.9 \\
\hline 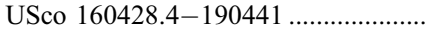 & USco $160428.0-190434$ & 1.04 & 0.27 & 9.28 & 1.02 & 0.31 & 11.01 & 9.77 & 321.3 \\
\hline USco 160707.7-192715 ................... & USco 160708.7-192733 & 0.95 & 0.24 & 9.80 & 1.00 & 0.29 & 11.17 & 23.45 & 140.4 \\
\hline 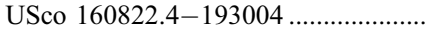 & USco $160823.2-193001$ & 0.97 & 0.18 & 9.06 & 1.11 & 0.27 & 9.47 & 13.47 & 71.4 \\
\hline 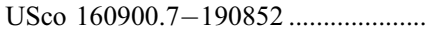 & USco $160900.0-190836$ & 1.07 & 0.32 & 9.15 & 1.01 & 0.38 & 10.96 & 18.92 & 326.5 \\
\hline USco $161010.4-194539$.................... & USco $161011.0-194603$ & 0.97 & 0.28 & 10.41 & 0.96 & 0.33 & 11.38 & 25.59 & 160.8 \\
\hline 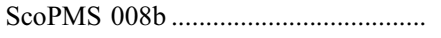 & ScoPMS 008a & 0.97 & 0.32 & 9.33 & 1.03 & 0.38 & 9.77 & 25.61 & 68.6 \\
\hline
\end{tabular}

wide-field images were used to create reference PSFs for each science target, while the postage stamp images have been used to identify close visual companions. The width of the wide-field images $\left(510^{\prime \prime}\right)$ corresponds to the width of each 2MASS survey tile; any image with larger width would include data taken at different epochs and therefore with different seeing conditions. The height was chosen to allow for $\gtrsim 10$ PSF reference stars brighter than $K \sim 11$ in all fields. The size of the overlap region between adjacent tiles was $60^{\prime \prime}$ in right ascension and $8.5^{\prime}$ in declination, so each science target appeared to be $>30^{\prime \prime}$ away from the edge in at least one tile.
The 2MASS images were produced by co-adding multiple exposures taken in sequence, each offset by $\sim 85^{\prime \prime}$ in declination, so drawing PSF reference stars from several arcminutes away could lead to nonuniform images. Only sources $\lesssim 40^{\prime \prime}$ north or south of a science target were observed in all six exposures that the science target was observed, and sources $\gtrsim 500^{\prime \prime}$ north or south do not share any simultaneous scans. However, all of the scans that contribute to a wide-field image were observed within $\sim 30 \mathrm{~s}$. We do not expect the seeing-based PSF to change on this short timescale, and we have found that the PSF is usually constant over each entire wide-field image $\left(\sigma_{\mathrm{FWHM}} \sim 0.1^{\prime \prime}\right)$. 


\subsection{Data Reduction and Source Identification}

We identified candidate companions and measured their fluxes from the postage stamp image of each sample member using the IRAF $^{2}$ package DAOPHOT (Stetson 1987), specifically with the PSF fitting photometry routine ALLSTAR. The template PSFs for each postage stamp image were created using the PSTSELECT and PSF tasks. We selected template stars for each source from the corresponding wide-field image; each PSF was based on the eight brightest, unsaturated stars that appeared to be isolated under visual inspection. The appropriate photometric zero point was extracted from the image headers. We compared PSF fitting magnitudes for single stars to the corresponding PSC values in order to test our results; there is no systematic offset, and the standard deviation of the random scatter in $m_{\mathrm{PSF}}-m_{\mathrm{PSC}}$ is $\sim 0.03$ mag.

As we have discussed in previous publications (Kraus et al. 2005, 2006), one limitation of ALLSTAR-based PSF photometry is that binaries with very close ( $\left.\leqslant \theta_{\mathrm{FWHM}}\right)$ separations are often not identified, even when their combined PSF deviates significantly from that of a true point source. This limitation can be overcome for known or suspected binaries by manually adding a second point source in approximately the correct location and letting ALLSTAR recenter it to optimize the fit. However, this method requires objective criteria for identifying suspected binaries; subjective selection methods like visual inspection would not allow us to rigorously choose and characterize a statistically complete sample. We have found that ALLSTAR's $\chi^{2}$ statistic, which reports the goodness of fit between a source and the template PSF, is an excellent diagnostic for this purpose. Since there are images in three bandpasses, we use a single diagnostic value, denoted $\chi_{3}$, which is the sum of the three $\chi^{2}$ values obtained for each association member when fitted with a single point source. We list the value of $\chi_{3}$ for each association member in Table 2.

In Figure 1 we plot the values of $\chi_{3}$ as a function of $K$-band magnitude for a subset of sample members with no known companions between $0.5^{\prime \prime}$ and $15^{\prime \prime}$ (according to the surveys of Leinert et al. 1993; Ghez et al. 1993; Simon et al. 1995; Duchene 1999; Kohler et al. 2000; Kraus et al. 2005, 2006; R. White et al. 2007, in preparation). The goodness of fit degrades rapidly for saturated stars $(K \lesssim 8)$, so our technique does not discriminate between single stars and candidate binaries in this regime. However, since there are few stars brighter than the saturation limit, we decided not to reject them until we were certain we could not identify any binary systems via other methods. The distribution of $\chi_{3}$ values for unambiguously unsaturated stars $(K>8.5)$ is not normally distributed, but $95 \%$ of these stars produce fits with $\chi_{3}<2.5$, so we have selected all sources with $\chi_{3} \geq 2.5$ as candidate binary systems.

The mean value of $\chi_{3}$ for single stars should be $\sim 3$ since it represents the sum of three variables that follow a $\chi^{2}$ distribution. However, we find that the mean value reported by ALLSTAR for unsaturated single sources is $\sim 1.75$. This disagreement is caused by an overestimate of the photometric errors in each observation by ALLSTAR. The co-adding and subsampling process used in the 2MASS image processing pipeline results in correlated noise between adjacent pixels of the final survey images, so the true uncertainties are lower than those estimated solely by Poisson statistics (Skrutskie et al. 2006).

We identified the candidate binaries in our sample based on this empirically motivated $\chi_{3}$ selection criterion, and then we

\footnotetext{
2 IRAF is distributed by the National Optical Astronomy Observatory, which is operated by the Association of Universities for Research in Astronomy, Inc., under cooperative agreement with the National Science Foundation.
}

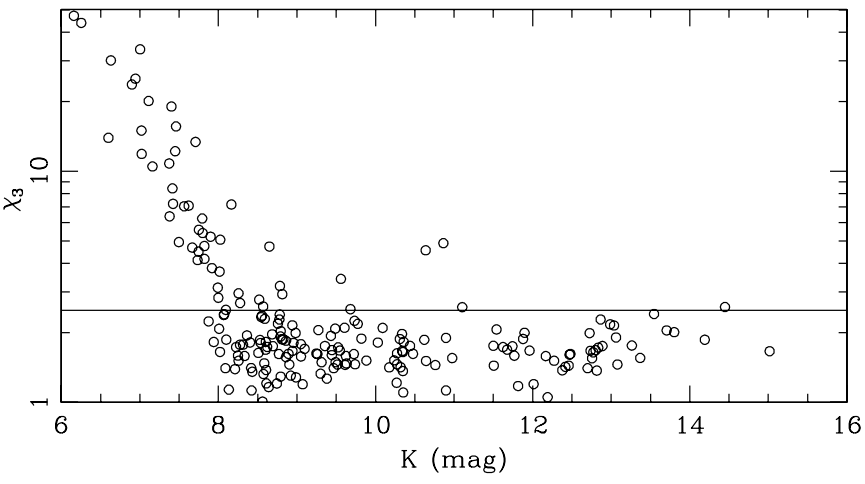

FIG. 1.-Plot of the goodness of fit as a function of $K$-band magnitude for 203 objects with no wide companions $\left(0.5^{\prime \prime}-15^{\prime \prime}\right)$. The sharp increase in $\chi_{3}$ at $K \sim 8$ is due to the onset of image saturation; the stars in this brightness range are typically late $\mathrm{K}$ or early $\mathrm{M}$, so saturation begins simultaneously in all three bands. The solid line at $\chi_{3}=2.5$ denotes the $95 \%$ confidence interval for nominally single stars; we have selected all sample members above this limit as candidate close binaries. We found that our fitting algorithm for identifying companions is effective for mildly saturated stars, so we include association members up to $K=6$.

attempted to fit each with a pair of point sources separated initially by the PSF FWHM (3") and with position angle corresponding to the angle of maximum elongation of the system PSF. The ALLSTAR routine optimized the components' separation, position angle, and magnitudes to produce the optimal fit; as we further summarize in $\S 3.3$, known binaries were typically fitted with consistent positions and flux ratios in all three bandpasses, while contaminants (such as sources with an erroneous template PSF in one filter) did not produce consistent fits in multiple images. We adopt the criterion that any candidate binary with component positions within $1^{\prime \prime}$ ( $3 \sigma$ for astrometry of very close, faint companions; $\S 3.4$ ) in all three filters is a bona fide visual binary. We found that saturated stars produced fits for erroneous companions at separations of $1.0^{\prime \prime}-1.5^{\prime \prime}$, so we have rejected all candidate companions to saturated targets $\left(K_{\text {tot }}<8\right)$ with separations of $<2^{\prime \prime}$. Known binaries with wider separations produced consistent fits even in the saturated regime for systems fainter than $K \sim 6$, so we adopted this as a maximum brightness limit for our sample.

Finally, we compared the location of each candidate companion with the online catalog of 2MASS image artifacts. We found that a candidate companion to MHO Tau 4 was coincident with a persistence artifact flag. Furthermore, a previous highresolution imaging survey with $H S T$ (Kraus et al. 2006) found no optical counterpart to a limit of $z^{\prime} \sim 24$, so we removed this candidate companion from further consideration and treat $\mathrm{MHO}$ Tau 4 as a single star.

\subsection{Sensitivity Limits}

We determined companion detection limits as a function of distance from the primary stars via a Monte Carlo simulation similar to that of Metchev et al. (2003). We used the IRAF task DAOPHOT/ADDSTAR to add artificial stars at a range of radial separations and magnitudes to the images of FO Tau, MHO Tau 5, KPNO Tau 8, and KPNO Tau 9. These four sources have been shown to be single to the limits of high-resolution imaging (Ghez et al. 1993; Kraus et al. 2006) and span the full range of brightness in this sample. We then attempted to identify the artificial companions via PSF fitting photometry. Our photometric routines attempt simultaneous source identification in all three filters in order to separate erroneous detections from genuine companions, so we created the same synthetic source in all three filters 


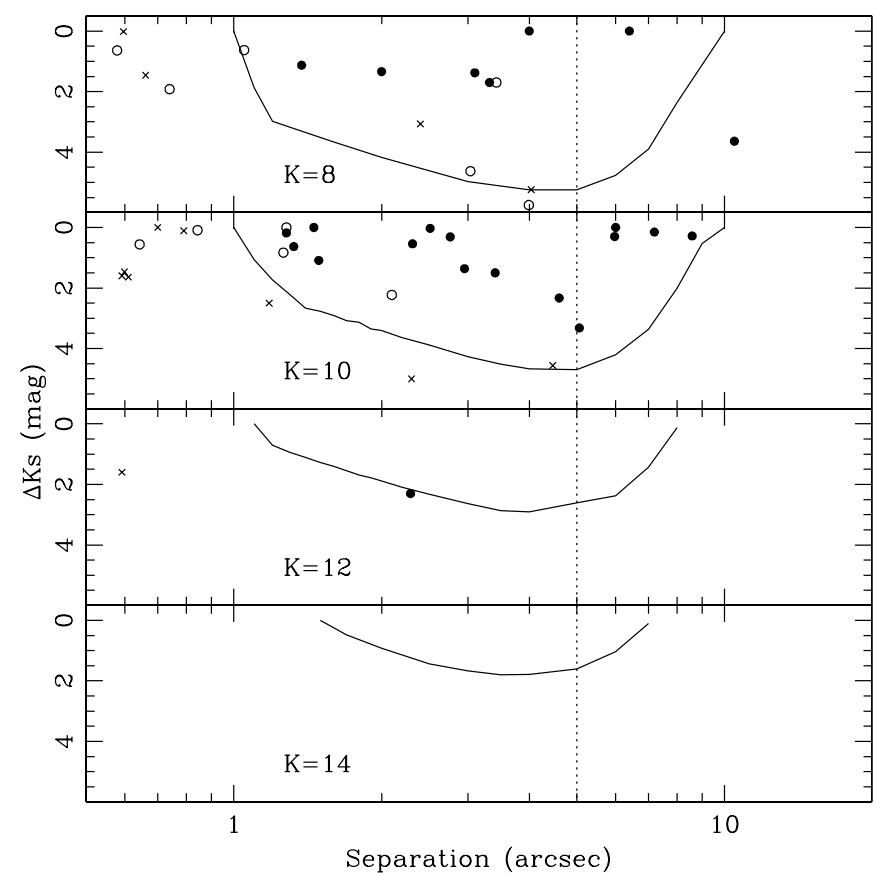

Fig. 2.-Detection frequencies as a function of separation for artificially introduced companions to four known single objects spanning the survey sample's brightness range: FO Tau $(K=8.12)$, MHO Tau $5(K=10.06)$, KPNO Tau 8 $(K=11.99)$, and KPNO Tau $9(K=14.19)$. The solid lines denote the $50 \%$ detection limit for our PSF fitting photometry. The symbols represent known binary companions from high-resolution $K$-band multiplicity surveys in Upper Scorpius (Kohler et al. 2000) and Taurus (R. White et al. 2007, in preparation and references therein). Filled circles denote companions that we recovered, open circles denote companions that passed our $\chi^{2}$ criterion but did not produce significant fits, and crosses denote companions that were not recovered. The dotted line shows the minimum separation at which the PSC will identify all companions bright enough to be considered in our search $(K<14.3)$.

using colors from the $2 \mathrm{Myr}$ Baraffe isochrones (Baraffe et al. 1998).

In Figure 2 we show our survey's 50\% detection limits as a function of separation for identifying candidate companions using the same PSF fitting algorithm as our actual search program. The minimum separation at which we can detect equal-flux companions is $\sim 1^{\prime \prime}$ for bright, unsaturated sources and $\sim 1.6^{\prime \prime}$ for sources just above our adopted $K$-band magnitude limit $(K=14.3)$. The $10 \%$ and $90 \%$ detection limits are typically $\sim 0.5$ mag below and above the $50 \%$ limit. The sensitivity of PSF fitting photometry falls at separations $\gtrsim 5^{\prime \prime}$ since objects become cleanly resolved and most companion flux falls outside the fitting radius for the primary. However, the PSC is complete to at least $K=14.3$ at larger separations, so wider companions will be recovered by our search of the catalog.

We also show the separation and flux ratio for known binary systems that have been detected in $K$-band surveys (Kohler et al. 2000; R. White et al. 2007, in preparation) and whether these systems were unambiguously recovered (via either PSF fitting photometry or the PSC), identified as candidate systems based on the $\chi_{3}$ criterion, or not recovered. The limits between detected and nondetected systems are roughly consistent with our empirically determined magnitude limits, but there are few known systems that fall near these limits. There are only two known wide systems among the faintest members of our sample $(K>11)$, so we cannot significantly test the detection limits of our search method in this brightness range. However, we identified four additional candidate companions to sources in this brightness range, plus numerous likely background stars, so our survey appears to be sensitive to companions in this regime.

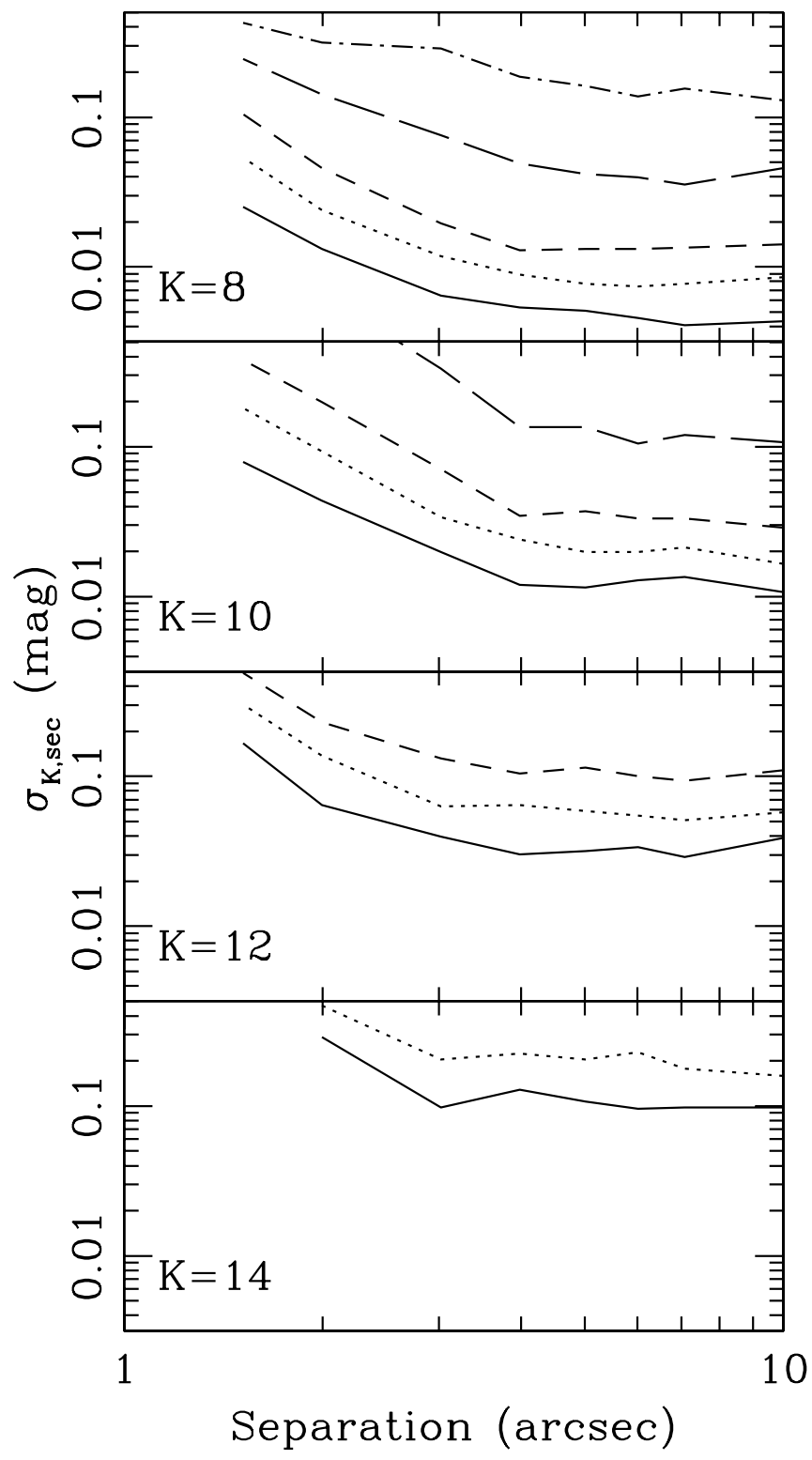

FIG. 3.-Uncertainty in the measured binary companion brightness as a function of separation for simulated binary images spanning the range of primary and secondary brightnesses. The flux ratios shown are $\Delta K=0,1,2,4$, and 6 (solid, dotted, short-dashed, long-dashed, and dot-dashed lines, respectively). The photometric uncertainties increase sharply at separations of $\$ 3^{\prime \prime}$, suggesting that observed photometric colors will not be accurate in this separation range.

\subsection{Uncertainties in Binary Properties}

Many of our candidate binaries have separations of $\leqslant \theta_{\text {FWHM }}$, so our measurements could be subject to significant uncertainties. We tested these uncertainties by using a Monte Carlo routine to produce synthetic images for binaries spanning a range of primary brightnesses, flux ratios, and separations. Specifically, we used ADDSTAR to construct simulated JHK images, and then we measured the binary fluxes and separations for each set of simulated images using ALLSTAR. For each combination of parameters, we produced 100 sets of synthetic images with randomly distributed position angles. The $J-K$ and $H-K$ colors for the secondaries were drawn from the $2 \mathrm{Myr}$ isochrone of Baraffe et al. (1998) in order to determine realistic values for $\Delta K, \Delta H$, and $\Delta J$.

In Figure 3 we show the standard deviation in the measured brightness for our simulated binary companions as a function of 


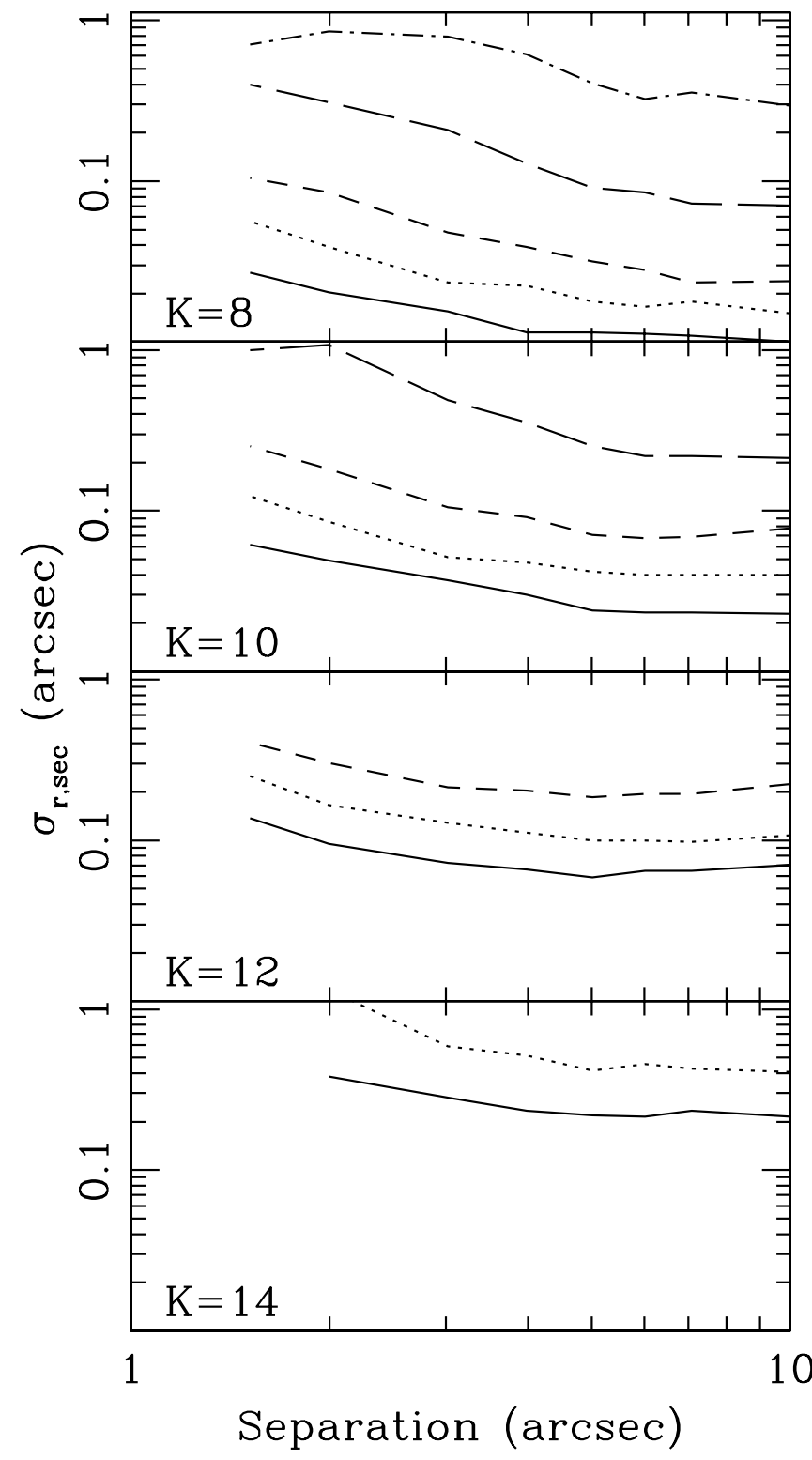

FIG. 4.-As in Fig. 3, showing uncertainties in binary secondary positions as a function of separation.

separation. These simulations predict that photometric uncertainties increase significantly at separations of $\leqslant 3^{\prime \prime}$, so measured colors may not be reliable at small separations. As we describe in $\S 3.5$, these colors are necessary at large separations $\left(>5^{\prime \prime}\right)$ to distinguish candidate companions from background stars. However, contamination from background sources should be low at small separations $\left(\leqslant 3^{\prime \prime}\right)$ due to their low surface density, so we can neglect these selection criteria with only a minor increase in the number of erroneous binary identifications.

In Figure 4 we show a similar plot of the rms scatter in the measured position of the secondary. The typical standard deviations are $\lesssim 0.3^{\prime \prime}$ for all but the faintest companions, so the uncertainties in our measured separations should have similar precision. Given these positional uncertainties, the corresponding uncertainties in position angles range from $1^{\circ}$ to $10^{\circ}$, depending on the binary separation. The standard deviations in secondary position for our simulated images are consistent with the scatter between the three filters for each observed binary, so we adopt the results from these simulations as our estimated uncertainties.
We also conducted Monte Carlo tests to determine the probability of mistakenly identifying a true single star as a binary. We constructed a series of simulated images (100 each for four objects spanning our sample's range of brightness) and then tried to fit each object with two point sources. We found that this never produced consistent fits in three filters, although faint peaks due to noise were occasionally identified in one of the three images. This suggests that the probability of an erroneous binary identification due to statistical errors is low $(<1 \%)$. This agrees with our results for known single stars; as we note in $\S 3.2,5 \%$ of known single stars fall above our $\chi_{3}$ criterion for identifying candidate binaries. However, none of these yielded fits for multiple point sources in all three filters.

\subsection{Field Star Contamination}

The identification of binary companions based solely on proximity is complicated by contamination from foreground dwarfs, background giants, and reddened early-type background dwarfs. We have not conducted follow-up spectroscopic or astrometric observations to confirm association membership, so we must limit the survey to a total area in which the contamination from background stars is small compared to the number of candidate binary companions. We estimate the surface density of contaminants for each association based on the total number of objects within an annulus of $30^{\prime \prime}-90^{\prime \prime}$ from all of the association members in our sample. Field surveys (e.g., Duquennoy \& Mayor 1991; Reid \& Gizis 1997) have identified few binaries with projected separations of $\gtrsim 500 \mathrm{AU}\left(~ \gtrsim 30^{\prime \prime}\right.$ at the distance of our sample members), so this method will also address the probability of chance alignment with other association members.

Our estimate of the contamination could be influenced by variations in background source counts due to the large angular extent of these associations or by variations in galactic latitude or extinction. The result would be a systematic overestimation of the association probability for candidate companions at points of high contamination and a corresponding underestimation at points of low contamination. However, any local deviation from the mean contamination rate should not affect the binary statistics for the association as a whole since the ensemble background at $30^{\prime \prime}-90^{\prime \prime}$ will match the ensemble background at $<30^{\prime \prime}$. Our subsequent cuts against color, mass ratio, and separation will also help to homogenize the sample by preferentially removing background stars.

Most previous multiplicity surveys were based on observations in a single optical or near-infrared bandpass (e.g., Kohler et al. 2000); in the absence of color information, these surveys can only estimate physical association probabilities for candidate companions based on the surface density of background stars of similar brightness. Since 2MASS includes images in three filters, we can reject most background stars by requiring colors consistent with regional membership ( $\S 4.1)$. Specifically, we have plotted $(K, J-K)$ and $(K, H-K)$ color-magnitude diagrams (CMDs) for each region, and we require prospective binary companions to fall above a smoothed field main sequence (Bessell \& Brett 1988; Leggett et al. 2001) for the regional distance in both CMDs. We have chosen to use $K$ as a proxy for luminosity instead of $J$ in order to minimize the effect of extinction for background stars. This choice will cause disk-bearing association members to sit preferentially higher in our CMDs, but this moves them farther from our selection cutoff, so our results should be robust.

As a test of these color criteria, we have plotted CMDs for the members of our primary sample. We find that $\sim 97 \%$ of the primaries have colors consistent with our definition of association membership, so any incompleteness in the selection of binary 

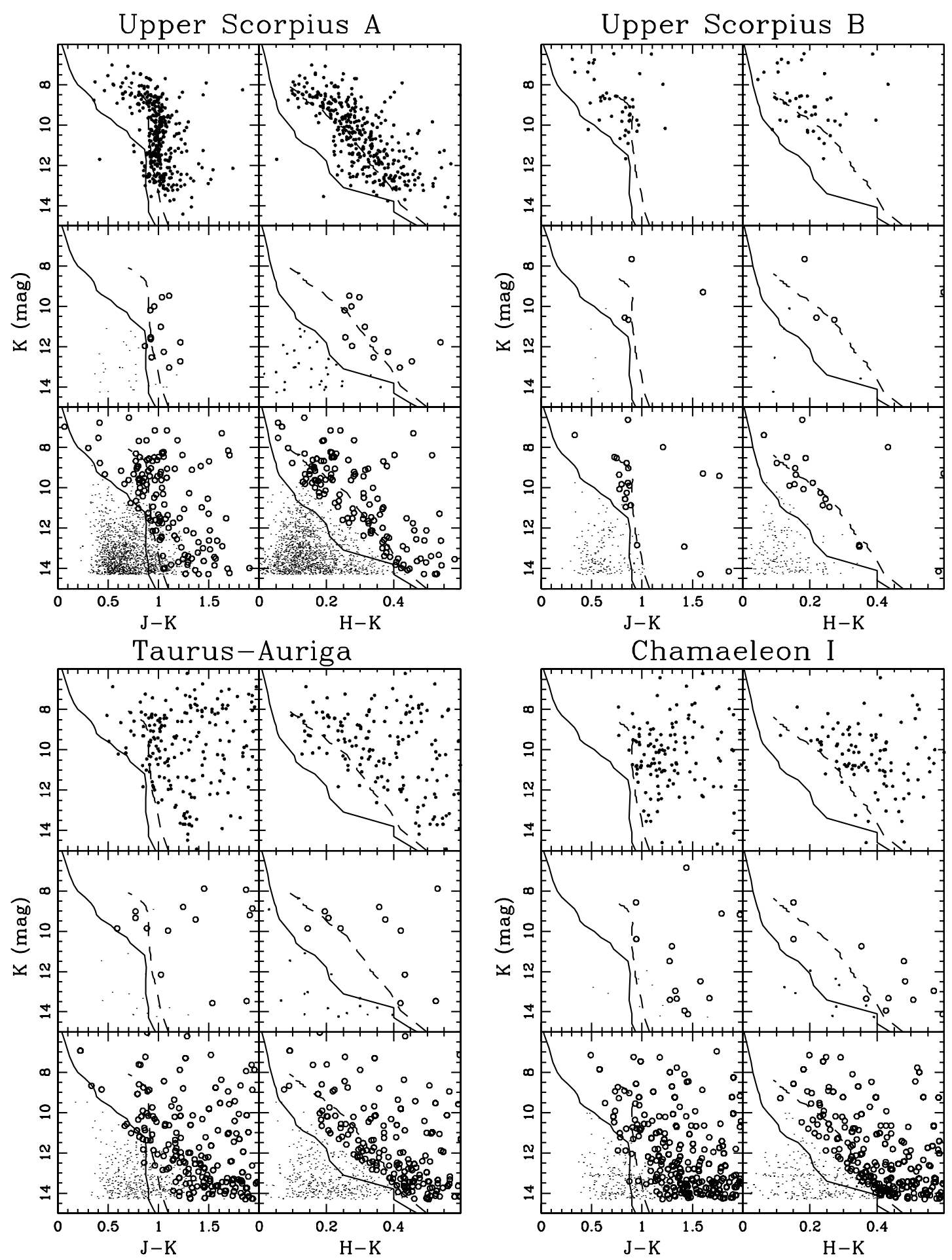

FIG. 5.-K, $J-K$ and $K, H-K$ CMDs for the four regions in our survey. The top panels show the confirmed association members in our survey, the middle panels show all objects within $5^{\prime \prime}-15^{\prime \prime}$ of known association members, and the bottom panels show all objects within the background annuli $\left(30^{\prime \prime}-90^{\prime \prime}\right)$. The solid line shows the main sequence at the association distance, and the dashed line shows the isochrone for the adopted association age (Table 2). In the top panels, association members are shown with filled circles. In all other panels, sources that lie above a smoothed main sequence in both CMDs are shown with open circles, and other sources are shown with small dots.

companions should be negligible. Most unselected primaries fall just below our color cuts; the only sample members that fall well below the association sequences are GSC 06191-00552 and USco 160803.6-181237. Both of these objects are claimed to be spectroscopically confirmed members of USco-A, but the spectra are not available in the literature. We have not detected any binary companions to these objects, so their erroneous inclusion in our sample would not significantly change our results. However, it might be prudent to reconsider their membership status with additional spectroscopic observations in the future.

\section{RESULTS}

\subsection{Candidate Binary Companions}

We identified a total of 451 well-resolved visual companions brighter than $K=14.3$ within $30^{\prime \prime}$ of our sample members in the 
TABLE 4

Association Star Counts

\begin{tabular}{|c|c|c|c|c|c|c|c|c|c|c|c|c|}
\hline \multirow[b]{2}{*}{$\begin{array}{c}\text { Separation } \\
(\operatorname{arcsec})\end{array}$} & \multicolumn{3}{|c|}{ Chamaeleon $\mathrm{I}\left(N=147^{\mathrm{a}}\right)$} & \multicolumn{3}{|c|}{ Taurus-Auriga $\left(N=235^{\mathrm{a}}\right)$} & \multicolumn{3}{|c|}{$\mathrm{USCO}_{\mathrm{C}} \mathrm{A}\left(N=356^{\mathrm{a}}\right)$} & \multicolumn{3}{|c|}{$\mathrm{USCO}_{\mathrm{C}} \mathrm{B}\left(N=45^{\mathrm{a}}\right)$} \\
\hline & $\begin{array}{l}\text { Source } \\
\text { Count }^{b}\end{array}$ & $\begin{array}{l}\text { Color } \\
\text { Valid }^{\mathrm{b}}\end{array}$ & $\begin{array}{l}\text { Bkgd } \\
\text { Stars }\end{array}$ & $\begin{array}{l}\text { Source } \\
\text { Count }\end{array}$ & $\begin{array}{l}\text { Color } \\
\text { Valid }\end{array}$ & $\begin{array}{l}\text { Bkgd } \\
\text { Stars }\end{array}$ & $\begin{array}{l}\text { Source } \\
\text { Count }\end{array}$ & $\begin{array}{l}\text { Color } \\
\text { Valid }\end{array}$ & $\begin{array}{l}\text { Bkgd } \\
\text { Stars }\end{array}$ & $\begin{array}{l}\text { Source } \\
\text { Count }\end{array}$ & $\begin{array}{l}\text { Color } \\
\text { Valid }\end{array}$ & $\begin{array}{l}\text { Bkgd } \\
\text { Stars }\end{array}$ \\
\hline $0-3^{\mathrm{c}} \ldots \ldots \ldots \ldots \ldots \ldots$ & 7 & $\ldots$ & 0.9 & 9 & $\ldots$ & 0.9 & 15 & $\ldots$ & 2.0 & 8 & $\ldots$ & 0.3 \\
\hline $3-5 \ldots \ldots \ldots \ldots \ldots \ldots$ & 5 & 5 & 1.0 & 6 & 5 & 0.7 & 8 & 4 & 0.3 & 1 & 0 & 0.1 \\
\hline $5-10 \ldots \ldots \ldots \ldots \ldots \ldots$ & 8 & 6 & 4.8 & 10 & 5 & 3.1 & 12 & 5 & 1.4 & 6 & 4 & 0.4 \\
\hline $10-15 \ldots \ldots \ldots \ldots \ldots$ & 19 & 12 & 8.0 & 22 & 11 & 5.2 & 32 & 8 & 2.4 & 3 & 0 & 0.6 \\
\hline $25-30 \ldots \ldots \ldots \ldots \ldots . . . . .$. & 39 & 28 & 17.6 & 33 & 16 & 11.4 & 60 & 5 & 5.3 & 9 & 4 & 1.3 \\
\hline $30-90 \ldots \ldots \ldots \ldots \ldots . . .$. & 766 & 461 & $\ldots$ & 733 & 298 & $\ldots$ & 1566 & 138 & $\ldots$ & 215 & 34 & $\ldots$ \\
\hline
\end{tabular}

a The total sample size for each region, as summarized in Table 1.

b The number of unassociated contaminants was estimated from the surface density of sources that meet our color selection criteria in the $30^{\prime \prime}-90^{\prime \prime}$ separation range; most of these sources should be foreground stars, background stars, or unbound association members.

${ }^{c}$ We cannot use color criteria at separations of $<3^{\prime \prime}$ due to the poor photometric precision $(\S 3.4)$, so the surface density of unassociated contaminants is higher.

2MASS PSC ( $(3.1)$, as well as 48 close $\left(\lesssim 5^{\prime \prime}\right)$ candidate companions based on our PSF fitting photometry of 2MASS image data $(\S 3.2)$. We have chosen $30^{\prime \prime}(\sim 5000 \mathrm{AU})$ as an absolute upper limit for identifying candidate companions since it corresponds to the maximum separation seen for field binaries at the distances of these association members. We also found $3280 \mathrm{vi}-$ sual companions within $30^{\prime \prime}-90^{\prime \prime}$ of our sample members. Since the ratio of sources at $0^{\prime \prime}-30^{\prime \prime}$ and $30^{\prime \prime}-90^{\prime \prime}$ is roughly equal to the ratio of areas $(1: 8)$, we expect that most of the sources within $30^{\prime \prime}$ of our sample stars are foreground or background stars having colors inconsistent with association membership.

In Figure 5 we present $(K, J-K)$ and $(K, H-K)$ CMDs for the four regions showing all confirmed association members in our sample and all companions in two separation ranges $\left(5^{\prime \prime}-15^{\prime \prime}\right.$ and $30^{\prime \prime}-90^{\prime \prime}$ ) corresponding to likely companions and likely background stars. We summarize the number of objects that pass or fail the color selection criteria $(\S 3.5)$ as a function of separation in Table 4. We also estimate the number of contaminants that are expected to pass both selection criteria in each separation range, assuming that the source density at $30^{\prime \prime}-90^{\prime \prime}$ represents the contaminant source density.

We showed in $\S 3.4$ that the uncertainties in our PSF fitting photometry become significant at small separations, so we cannot use color criteria to identify candidate companions inside $\sim 3^{\prime \prime}$. However, given the low surface density of background sources and the faintness of most nonmembers, we expect only a small level of contamination in this separation range. Each of the 39 candidate companions at separations $<3$ " has a sufficiently high probability of physical association ( $\gtrsim 80 \%)$ to merit inclusion in our sample without using color cuts.

We have defined the maximum separation at which we identify candidate binary companions by requiring that the number of sources that pass our color selection requirement in each separation bin be $\gtrsim 2$ times the number of expected background companions. The corresponding probability that any individual source inside that separation limit is a background star will be $\$ 50 \%$. Based on the expected contamination rates and visual companion counts in Table 4, these separations are $10^{\prime \prime}$ for ChamI, $15^{\prime \prime}$ for Taurus, $20^{\prime \prime}$ for USco-A, and $30^{\prime \prime}$ for USco-B. The separation limit is lower for regions with higher extinction since a higher fraction of background stars are reddened into our selection range. We adopt these separation limits as our criteria for identifying candidate binary companions. We note that sources at higher separations still have a nonnegligible probability of association, but the probability that any individual source is a binary companion will be low.

Using the color and separation cuts described above, we have identified (of 451 sources identified in the PSC and 48 sources identified with PSF fitting photometry) a total of 18 candidate binary companions in ChamI, 32 in Taurus, 40 in USco-A, and 17 in USco-B. Of these candidates, $4,7,23$, and 5, respectively, have not been previously reported in the literature. We summarize the binary properties of these candidate systems in Table 5 . Some of the very wide and very faint companions are likely to be unassociated foreground or background stars, so we consider a restricted range of separations and mass ratios in our subsequent

TABLE 5

Candidate Wide Binary Systems

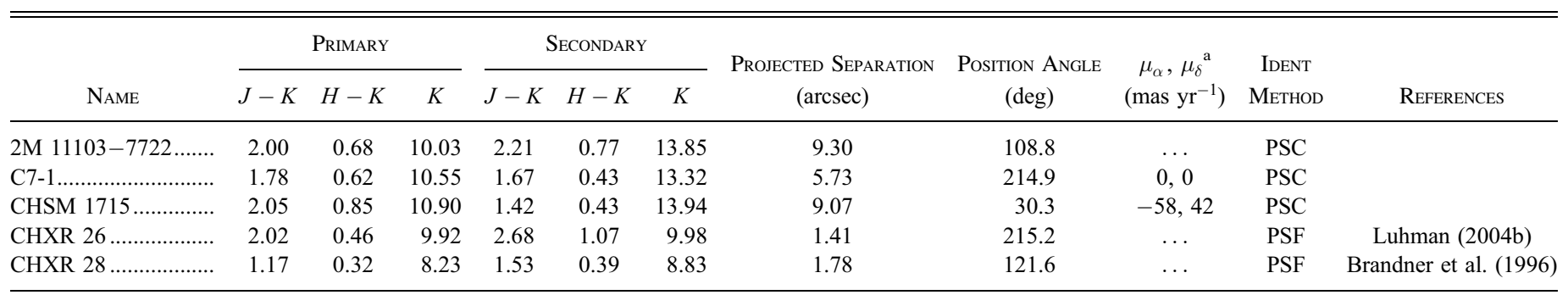

\footnotetext{
Notes.-Table 5 is published in its entirety in the electronic edition of the Astrophysical Journal. A portion is shown here for guidance regarding its form and content.

a An entry of 0,0 denotes a source that was detected by the USNO-B survey but did not show a significant proper motion. Ellipses denote a source that was not detected by the USNO-B survey.
} 
TABLE 6

Ultrawide Visual Companions

\begin{tabular}{|c|c|c|c|c|c|c|c|c|c|c|}
\hline $\mathrm{C} 1-6 \ldots \ldots \ldots \ldots \ldots \ldots$ & 3.92 & 1.68 & 8.67 & 2.26 & 0.70 & 13.75 & 24.51 & 123.8 & $\ldots$ & OTS 14 (candidate; Oasa et al. 1999) \\
\hline Cam 2-19 ........... & 2.40 & 0.74 & 10.25 & 2.36 & 0.72 & 13.45 & 23.13 & 107.6 & $\ldots$ & $\ldots$ \\
\hline Cam 2-42 ........... & 2.44 & 0.73 & 9.16 & 2.09 & 0.50 & 13.51 & 27.64 & 261.7 & $\ldots$ & $\ldots$ \\
\hline Cam $2-42 \ldots \ldots \ldots .$. & 2.44 & 0.73 & 9.16 & 2.11 & 0.65 & 14.14 & 28.18 & 180.4 & $\ldots$ & $\ldots$ \\
\hline
\end{tabular}

Notes.-Table 6 is published in its entirety in the electronic edition of the Astrophysical Journal. A portion is shown here for guidance regarding its form and content.

statistical analysis. In Table 6 we list the other visual companions with separations $<30^{\prime \prime}$ (but wider than the association's companion identification limit) that have colors consistent with association membership and separations greater than the limits given above. Many of these sources are expected to be background stars, but additional information (such as optical photometry or kinematic data) could be used in the future to remove additional contaminants and more securely identify any ultrawide binary companions.

\subsection{Previous Observations}

Many of our candidate companions have been identified previously in the literature, but as we note in Tables 5 and 6, several of our candidates have also been rejected as association members based on the absence of spectroscopic signatures of youth. Some of the candidates we list have probably been considered and rejected in previous work, but most surveys do not publish their catalog of confirmed field stars, so we cannot assess this number.

We also find that five members of our sample (USco 160700.1203309, SCH 16151115-24201556, and USco 80 in USco-A; 2MASS J04080782+2807280 and 2MASS J04414489+2301513 in Taurus) have candidate companions that are significantly brighter and thus are likely to be the system primary (making the known association member a binary secondary). This result is not surprising for the three Upper Sco members. Upper Sco is thought to contain several thousand low-mass members, and photometric surveys have identified many more candidates than could be confirmed via spectroscopy, so there are many more association members awaiting discovery. The two Taurus members are located on the edges of the association and were discovered by the only survey that considered these areas (Luhman 2006). Our newly identified candidate companions are both brighter than the upper brightness limit for this survey $(H=10.75)$, so there were no previous opportunities for them to have been discovered.

Finally, we find that five candidate companions identified in previous surveys have $2 \mathrm{MASS}$ colors inconsistent with association membership: UX Tau B, V819 Tau B, HBC 355 (HBC 354 B), RX J1524.2-3030B, and RX J1559.8-2556B. Since $\sim 3 \%$ of the spectroscopically confirmed association members in our primary star sample did not meet both color cuts, we expect (adopting the same percentage for the secondaries) that only $\sim 1-2$ bona fide binary companions would not be selected. However, close pairs of stars have larger photometric errors, which increases the probability that some companions might fall outside our selection cuts. Of these five companions, three fall just below the color cuts (UX Tau B, HBC 355, and RX J1524.23030B) in our CMDs and the other two fall significantly below the color cuts, so we suggest that the first three are erroneous re- jections, and therefore we keep these objects, while we consider the other two to be valid rejections. ${ }^{3}$

\subsection{Inferred Stellar Properties}

In Table 2 we list the inferred spectral types and masses for all of the association and cluster members in our sample. Spectral types are taken from the primary reference and were typically determined via low- or intermediate-resolution spectroscopy. We assume that the spectroscopically determined spectral type and mass for previously unresolved binary systems correspond to the primary mass and spectral type. Equal-mass binary components should have similar spectral types, and the flux from unequal-mass systems should be dominated by the primary; in either case, spectroscopic observations of the unresolved system should have been affected only marginally by the flux from the secondary.

We estimated the masses of sample members by combining mass-temperature and temperature-spectral type relations from the literature. No single set of relations spans the entire spectral type range of our sample, so we have chosen the M dwarf temperature scale of Luhman et al. (2003b), the early-type ( $\leq \mathrm{M} 0)$ temperature scale of Schmidt-Kaler (1982), the high-mass stellar models of D'Antona \& Mazzitelli (1997), and the low-mass stellar models of Baraffe et al. (1998; NextGen). We apply the D’Antona \& Mazzitelli (1997) mass-temperature models for masses of $\gtrsim 1 M_{\odot}$ and the NextGen models for masses of $\lesssim 0.5 M_{\odot}$; in the $0.5-1.0 M_{\odot}$ regime, we have adopted an average sequence. For each association, we adopt the models corresponding to the mean age listed in Table 1; this will introduce some uncertainty given the unknown age spread for each association. Large systematic errors may be present in these and all pre-main-sequence models (e.g., Baraffe et al. 2002; Hillenbrand \& White 2004; Close et al. 2005; Reiners et al. 2005), so they are best used for relative comparison only.

Much of the uncertainty in theoretical mass-temperature relations can be assessed in terms of a zero-point shift in the mass; preliminary observational calibrations by the above authors suggest that theoretical models overestimate masses by $10 \%-20 \%$ over most of our sample mass range. This suggests that theoretical predictions of relative properties (e.g., mass ratios, $q=m_{s} / m_{p}$ ) might be more accurate than absolute properties (e.g., individual component masses) since the systematic mass overestimates will cancel. Relative quantities are also largely independent of age and extinction, which are expected to be similar for binary components. We have combined our adopted mass-luminosity-spectral type

\footnotetext{
3 V819 Tau B has also been classified as a background star by Woitas et al. (2001) due to its position on a $(J, J-K) \mathrm{CMD}$ and by Koenig et al. (2001) due to an absence of X-ray emission. UX Tau B and HBC 355 are spectroscopically confirmed cluster members, and no membership assessments are available for the other two sources.
} 
TABLE 7

InFERREd Binary Properties

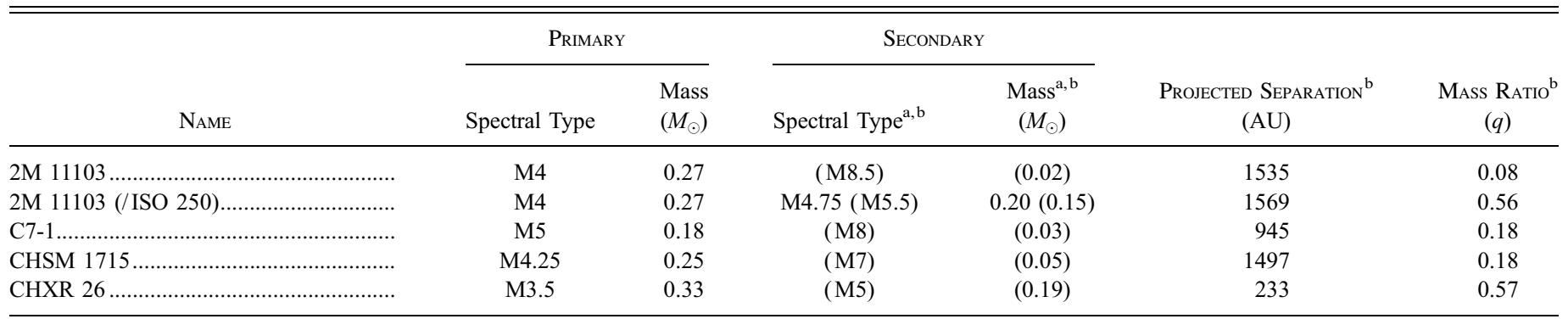

Notes.- Table 7 is published in its entirety in the electronic edition of the Astrophysical Journal. A portion is shown here for guidance regarding its form and content.

${ }^{a}$ Values in parentheses are estimated from the system flux ratio $\Delta J$ and the spectroscopically determined properties of the primary.

b Estimated statistical uncertainties are $\sim 10 \%$ for mass ratios, $\sim 20 \%$ for secondary masses, $\sim 2-3$ subclasses for spectral types, and $\sim 10 \%$ for projected separations.

relations with the near-infrared colors of Bessell \& Brett (1988) and the $K$-band bolometric corrections of Leggett et al. (1998, 2000,2002 ) and Masana et al. (2006) to predict values for $q$ as a function of primary brightness $m$ and flux ratio $\Delta m$ in all three 2MASS filters. Some of our sample members could possess $K$-band excesses due to hot inner disks, so we have adopted the $q$-values predicted by the $J$-band fluxes; this will not eliminate the effect but should minimize it. We have also combined our derived $q$-values with the estimated primary masses to predict secondary masses, and we use our mass-spectral type relations to predict the corresponding secondary spectral types.

We list the derived values for each binary system in Table 7 . Some wide binaries have independent spectral type determinations for both components, so we report derived quantities with parentheses and measured quantities without. The typical uncertainties in $q$ are $\sim 10 \%$ and represent the uncertainties in the photometry and the assigned spectral types, although some systematic effects (e.g., unresolved multiplicity or different levels of extinction) could produce far larger values. This can be seen in the discrepancies for some systems (e.g., GG Tau AB, MHO 2/1) that are known to be hierarchical multiple systems. We cannot quantify the unknown uncertainties in the theoretical models, but they should be considered when interpreting these results. The typical uncertainty in physical separation is $\sim 10 \%$ and reflects the uncertainty in angular separation and the unknown depth of each system in its association; we assume that each association has a total depth equal to its extent on the sky ( $\sim 40 \mathrm{pc}$ for Taurus and Upper Sco, $\sim 20 \mathrm{pc}$ for ChamI). The uncertainty in the mean association distance $(\sim 5 \mathrm{pc})$ introduces a systematic uncertainty of $\pm 3 \%$, but this is generally negligible.

\subsection{Binary Statistics}

Multiplicity surveys typically consider the frequency of binary systems for restricted ranges of parameter space (observed separations and mass ratios) corresponding to the survey completeness limits. For our analysis, we select a range of projected separations (330-1650 AU, set by the inner and outer detection limits of ChamI since those limits are most restrictive) and flux ratios ( $\Delta K<2$, corresponding to $q \gtrsim 0.25$ ) that should be complete for all but the lowest mass brown dwarfs in our sample. The inner separation limit and mass ratio limit are set by the resolution limit for low-mass sample members $(K \sim 12.3)$ in ChamI, while the outer separation limit is set by the background contamination in ChamI, where our mass ratio cut allows us to choose a $90 \%$ pure sample for separations $<10^{\prime \prime}$.

In Figure 6 we present plots of the wide binary frequency as a function of primary mass for each region in our sample. The binary fractions plotted correspond to our designated completeness regime: mass ratios $q>0.25$ and projected separations of 330-1650 AU. In the bottom panel, we show the field binary frequency in the same range of mass ratios and projected separations for solar-type stars (Duquennoy \& Mayor 1991), early to mid-M dwarfs (M0-M6; Reid \& Gizis 1997), and brown dwarfs (Bouy et al. 2003; Burgasser et al. 2003). We also show the corresponding frequencies for early-type stars in USco-A and USco-B (Kouwenhoven et al. 2005). The bin sizes were chosen to evenly sample the mass range of our survey $\left(0.025-2.50 M_{\odot}\right)$ for which the primary targets were brighter than our brightness cutoff $(K=$ 14.3). For each region in our survey, we also show the expected frequency for foreground and background sources that pass our color selection criteria and have $\Delta K<2$, assuming a background source count function $N(K)$ matching that shown in Figure 2; in all cases, the expected contamination rate is negligible. USco-A, ChamI, and Taurus all show a decline in the binary frequency with mass, consistent with the results shown for field multiplicity surveys. USco-B does not show a decline, but the uncertainties are not small enough to strongly constrain the slope of any mass dependence.

This binary search may not be complete for objects in the lowest mass bin where some binary companions could have been fainter than the survey detection limits $(K>14.3)$, so the true upper limits may be marginally higher. However, it has been observationally determined that most very low mass binaries in the field have mass ratios near unity $(q>0.7)$ and much smaller separations ( $\leqslant 20 \mathrm{AU})$, so we are unlikely to have missed any wider or lower mass ratio companions (Close et al. 2003; Burgasser et al. 2003; Bouy et al. 2003).

Another interesting distribution to consider would be the mass ratio distribution for wide binaries as a function of mass and environment. Unfortunately, extending our binary results along another axis of parameter space exceeds the statistical limits of our sample, leaving most bins with only zero to one detections. The best solution for this is to combine all regions into a single population. In Figure 7 we plot the mass ratio distribution in our survey separation range (330-1650 AU) for the three highest mass bins. We also show the best-fit distribution for solar-type stars in the field (Duquennoy \& Mayor 1991).

This result should be treated with caution since it represents an admixture of formation environments that likely does not match the composition of the field. As we show in Figure 6, the binary frequency appears to be fundamentally different in the dark cloud complexes (Taurus and Chamaeleon) than in USco-A. This distinction suggests that binary formation processes can vary significantly between different environments, and therefore 


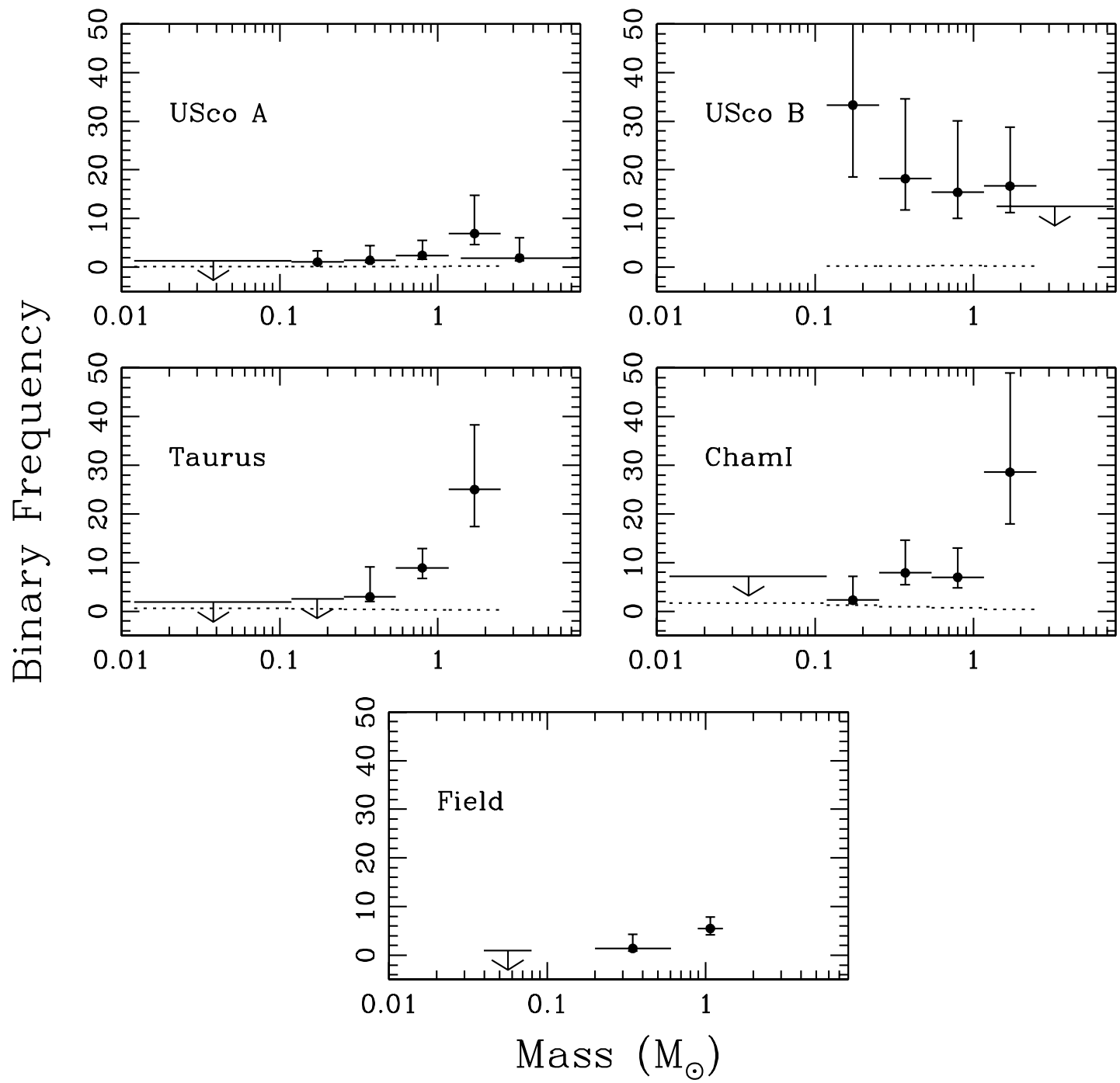

FIG. 6.-Wide (330-1650 AU) binary frequency as a function of mass for each region and as determined from field multiplicity surveys. The higher mass histogram bins are equally sized in $\log M$, but the three lowest mass bins have been combined to illustrate the absence of any companions. The error bars are calculated assuming binomial statistics. The highest mass data points for USco-A and USco-B denote the results of Kouwenhoven et al. (2005). The dashed lines show the expected frequency for each bin solely from foreground and background sources and unbound association members; they are not distinguishable from zero in most bins. Most upper limits for the lowest mass bins are also very close to zero.

that analysis of other binary properties should take the environment into account when possible.

\section{DISCUSSION}

\subsection{The Role of Mass and Environment in Multiplicity}

Field multiplicity surveys have established several apparent trends for the mass dependence of binary properties. Solar mass binaries occur at high frequency $(\gtrsim 60 \%)$ and possess high mean and maximum separations (30 and $10^{4} \mathrm{AU}$, respectively) and a mass ratio distribution biased toward low-mass companions ( $q<0.5$; e.g., Duquennoy \& Mayor 1991). By contrast, binaries near and below the substellar boundary occur at low frequency $(\sim 10 \%-20 \%)$ and possess small mean and maximum separations (4 and $20 \mathrm{AU}$, respectively) and a mass ratio distribution biased toward unity ( $q \gtrsim 0.7$; Close et al. 2003; Bouy et al. 2003; Burgasser et al. 2003). Observations of intermediate-mass M dwarfs (e.g., Fischer \& Marcy 1992; Reid \& Gizis 1997) suggest that their binary properties are transitional, with an intermediate binary frequency and possibly an intermediate separation range, plus a mass ratio distribution that is nearly flat for $q>0.1$.
These results have been supported by recent surveys of young open clusters and associations (e.g., Kohler et al. 2000; Patience et al. 2002; Luhman et al. 2005; Kraus et al. 2005, 2006; R. White et al. 2007, in preparation). High-mass stars in these regions typically have higher binary frequencies and wider binary separations than lower mass stars. There is emerging evidence that highdensity regions might have lower binary frequencies or preferentially smaller binary separations (e.g., Kohler et al. 2006), but it has not yet been conclusively determined whether this is a primordial feature or the result of early dynamical evolution.

\subsubsection{The Frequency of Wide Binary Formation}

Our results appear to be broadly consistent with the established paradigm of mass-dependent multiplicity. Wide (330-1650 AU) binaries are very common among stars of $\gtrsim 1 M_{\odot}$ and the frequency appears to decline smoothly with mass (Fig. 6). We found few wide binaries with primaries less massive than $\sim 0.25 M_{\odot}$. Wide binary systems also appear to be common in the low-density $\mathrm{T}$ associations (Taurus and ChamI) but comparatively rare in the USco-A OB association. This suggests that the trend against wide binaries in dense bound clusters might extend to unbound 


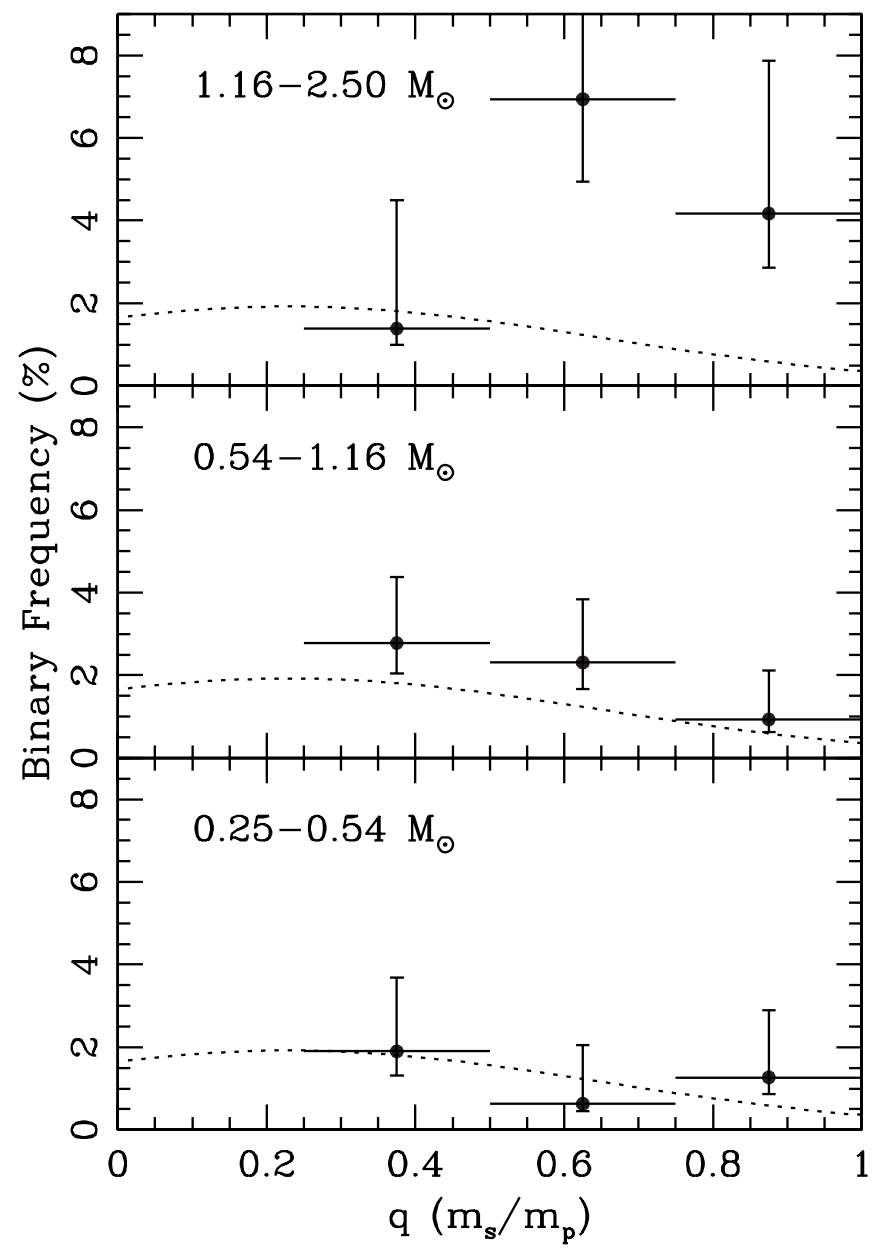

FIG. 7.- Mass ratio distribution for wide binaries in the three highest mass bins of our survey, calculated as a frequency among all sample members. The mass ratio distribution function found by Duquennoy \& Mayor (1991) for field solar-type stars is denoted with a dashed line. These results represent the sum over all associations in our sample; the binary frequency varies between environments (Fig. 6), and our sample represents a different admixture of formation environments than the field sample, so the sample and field frequencies should be compared with caution.

associations and therefore may be the result of another initial condition besides stellar density.

The high frequency of wide binary systems in USco-B also suggests that binary formation is not a pure function of stellar density. This population is kinematically associated with the Sco-Cen complex, and its proper motions most closely match the Upper Centaurus Lupus OB association, but the wide binary frequency for solar-type stars in USco-B is more consistent with the Tassociations in our sample. As we discuss further in Appendix C, this could also be explained if the stars in USco-B represent an evolved low-density association analogous to the $\rho$ Oph or Lupus complexes rather than a subgroup of an OB association.

\subsubsection{The Separation Distribution of Binary Systems}

The wide binary systems discovered by our survey only represent the outer tail of the separation distribution function. The measurement of its functional form will require large high-resolution imaging surveys sensitive to the core of the separation distribution $(\sim 10-100$ AU for solar-type stars, declining to $\sim 1-10 \mathrm{AU}$ for brown dwarfs). The uncertainties in results from the literature do not allow for strong constraints in this separation range, but our results are consistent with some of the proposed environmental trends. Wide binary systems appear to be significantly less common in USco-A than in USco-B, a fact that was noted by Kohler et al. (2000). Their high-resolution speckle interferometry survey found many binaries in USco-A with projected separations of 20-300 AU, but most of the binaries they discovered in USco-B had significantly higher separations. This led them to conclude that the binary separation distribution is biased toward tighter systems in USco-A than in USco-B. Numerous multiplicity surveys in Taurus and ChamI (e.g., Ghez et al. 1993, 1997) have also found a wider separation distribution than in USco-A, which is also consistent with our results.

Field multiplicity surveys have shown a probable mass dependence in the maximum binary separation. A census of previous surveys (Reid et al. 2001) found that the maximum field binary separation can be described empirically with an exponential function of the total system mass, $a_{\max } \propto 10^{3.3 M}$; an extension of this study to the substellar regime by Burgasser et al. (2003) found a corresponding quadratic function, $a_{\max } \propto M^{2}$. Burgasser et al. (2003) demonstrated, using the formalism of Weinberg et al. (1987), that this is not due to interactions with field stars or giant molecular clouds, but instead must be a feature of the formation process or a result of early dynamical evolution in the formation environment.

These empirical relations predict maximum separations of 330 and $1650 \mathrm{AU}$ for total system masses of $\sim 0.4$ and $0.6 M_{\odot}$, respectively. This prediction is consistent with the general minimum primary mass of $\sim 0.25 M_{\odot}$ that we have identified among the wide binaries in our sample. The implication is that this limit is indeed set by the T Tauri stage, either as a result of the formation process or during dynamical evolution while these systems are still embedded in their natal gas cloud.

However, we have identified one candidate system, USco 160611.9-193532, with an apparent low-mass primary $\left(0.13 M_{\odot}\right.$; spectral type M5) and a very wide projected separation (10.8"; $1550 \mathrm{AU})$. The USNO-B proper motion for the secondary

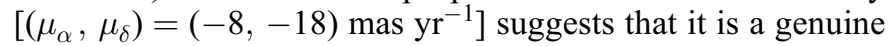
USco member and not a background star. As we will report in a future publication, subsequent observations with Laser Guide Star Adaptive Optics on the Keck II telescope also find that the primary is itself a close $\left(\sim 0.1^{\prime \prime}\right)$ equal-flux pair. If the wide visual companion is gravitationally bound, then this triple system $\left(M_{\text {tot }} \sim\right.$ $\left.0.4 M_{\odot}\right)$ does not follow the empirical mass-maximum separation relations. There are several other candidate wide binary systems that could potentially violate these relations, but the probability of background star contamination is high enough in these cases that association membership should be confirmed via spectroscopy before any conclusions are drawn.

Finally, a census of several star-forming regions by Simon (1997) found that pre-main-sequence stars tend to cluster on two length scales, with two-point correlation functions described by separate power laws. He concluded that small-scale clustering is a result of binary formation, while clustering on larger scales is a result of the condensation of multiple cores from single molecular clouds. This could potentially explain the excess of wide companions in Taurus, where the stars are younger and have not dispersed as far from their formation point. However, Simon (1997) found that the transition occurred at separations of $\sim 10^{4}$ AU in Taurus, and our survey truncates at $\sim 1500$ AU. This suggests that unless his initial estimate was significantly higher than the true transition point, all of our candidate companions fall within the binary regime.

\subsubsection{The Mass Ratio Distribution of Binary Systems}

Field multiplicity surveys have found that the mass ratio distribution varies significantly with primary star mass. Most solar mass primaries possess binary companions with low-mass ratios (Duquennoy \& Mayor 1991), early M dwarf primaries possess 
companions with a uniform mass ratio distribution (Fischer \& Marcy 1992), and late M dwarf and brown dwarf primaries possess companions with mass ratios near unity (e.g., Close et al. 2003; Siegler et al. 2005).

Our results cannot support any strong statistical claims, but they are largely consistent with this pattern. The only exception is that our results for the highest mass stars $\left(1.16-2.50 M_{\odot}\right)$ suggest the presence of a possible excess of wide similar-mass binaries. The excess is not consistent with background star contamination since the primaries are all very bright and most background stars should be significantly fainter; many of these binary companions have been confirmed independently as association members. It is also unlikely that we missed a significant number of companions with $0.25<q<0.50$; the detection limits (in $\Delta K$ or $q$ ) are best for bright stars and significantly exceed the limits of our statistically complete sample. This mass ratio distribution is also discrepant with that found by Kouwenhoven et al. (2005) for B-A stars in Sco-Cen; they found a distribution that is very similar to that of Duquennoy \& Mayor (1991), with a strong deficit of equal-mass binaries compared to unequal-mass binaries.

This discrepancy could represent another environmental dependence in multiple-star formation. Most of our high-mass binaries are found in the dark cloud complexes, while the Kouwenhoven sample was drawn from the three OB associations of Sco-Cen. The field sample of Duquennoy \& Mayor (1991) is probably also dominated by stars from dissolved OB associations or open clusters since those are thought to be the dominant star formation channel in our Galaxy. Since the binary frequency and binary separations appear to be affected by environmental conditions, it is plausible that the mass ratio distribution could also be affected.

\subsection{Summary of Implications for Wide Binary Formation}

Recent efforts to model binary formation have typically assumed that a cluster of 5-10 protostellar embryos form from a single fragmenting cloud core (e.g., Kroupa 1995; Sterzik \& Durisen 1998; Kroupa \& Bouvier 2003; Kroupa et al. 2003; Delgado-Donate et al. 2004; Hubber \& Whitworth 2005); these embryos would then undergo accretion and dynamical evolution to form single stars and stable multiple systems. This scenario would provide a convenient explanation for variations in binary properties between unbound associations or bound clusters since the stellar encounter rate would vary with stellar density.

However, the frequency of multiple stellar systems has been interpreted by Goodwin \& Kroupa (2005) to mean that the collapse and fragmentation of a cloud core produce only two or three stars, not 5-10. Larger systems would eject more single stars and tight binaries than are observed. Most models also predict that dynamical evolution would alter other stellar properties (spatial and velocity dispersion, disk lifetime, and accretion frequency), particularly at low masses. The preponderance of observational evidence shows that these properties are not consistent with strong dynamical evolution and do not vary significantly with mass (White \& Basri 2003; Luhman 2004c; White \& Hillenbrand 2004; Mohanty et al. 2005). However, some updated models of dynamical evolution suggest that not all of these features would show strong signatures (e.g., Bate \& Bonnell 2005).

The apparently minor role of dynamical processes suggests that binary properties might be established during the fragmentation of a cloud core, rather than in subsequent dynamical evolution. Sterzik et al. (2003) suggested that the initial cloud temperature could play a role in determining the frequency of wide binary systems. They noted that the radius of a cloud core at the end of isothermal collapse is inversely related to the initial cloud temperature. This suggests that regions of low temperature will have larger spatial scales during fragmentation and therefore a wider distribution of binary separations. This could be due to either a higher primordial cloud temperature or heating from other young stars.

We have found that the wide binary frequency in USco-A is comparable to that of open clusters like Praesepe or the Pleiades (Patience et al. 2002) or young clusters like IC 348 and the ONC (Luhman et al. 2005; Kohler et al. 2006). The stellar density is much lower in OB associations like USco-A, so the similarity between their binary populations suggests that another initial condition might play a key role in determining the binary separation distribution (and thus the frequency of wide binary systems). Kohler et al. (2006) also found that binary properties do not differ significantly between the core and the periphery of the $\mathrm{ONC}$, which span a significant range of stellar densities. These trends imply that wide binaries only form in the absence of high-mass stars, or equivalently that the presence of high-mass stars suppresses wide binary formation. The argument by Sterzik et al. (2003) could provide a natural explanation for this trend; highmass stars irradiate surrounding cloud cores, and the subsequent increase in temperature would decrease the final length scale over which fragmentation would occur.

\section{CONCLUSIONS}

We present the results of a search for wide binary systems among 783 members of three nearby young associations: Taurus-Auriga, Chamaeleon I, and the two subgroups of Upper Scorpius. This program analyzed near-infrared $J H K$ imagery from 2 MASS to search for wide $\left(1^{\prime \prime}-30^{\prime \prime} ; \sim 150-4500 \mathrm{AU}\right)$ companions to known association members, using color-magnitude cuts to reject likely background stars. We identified a total of 131 candidate binary companions in these associations, of which 39 have not been previously identified in the literature.

We find that the wide binary frequency (330-1650 AU; $q>$ 0.25 ) is a function of both mass and environment, with significantly higher frequencies among high-mass stars than lower mass stars and in the T associations than in the OB association. We discuss the implications for wide binary formation and conclude that the environmental dependence is not a direct result of stellar density or total association mass, but instead might depend on another parameter like the gas temperature of the formation environment.

We also analyze the mass ratio distribution as a function of mass and find that it largely agrees with the distribution seen for field stars. There appears to be a moderate excess of similar-mass $(q>0.5)$ wide binaries among the highest mass $\left(1.16-2.50 M_{\odot}\right)$ stars in our sample, but the number statistics do not support any other strong conclusions. The binary populations in these associations generally follow the empirical mass-maximum separation relation observed for field binaries, but we have found one candidate low-mass system (USco $160611.9-193532 ; M_{\text {tot }} \sim 0.4 M_{\odot}$ ) that has a projected separation $\left(10.8^{\prime \prime} ; 1550 \mathrm{AU}\right)$ much larger than the limit for its mass.

Finally, we find that the binary frequency in the USco-B subgroup is significantly higher than the USco-A subgroup and is consistent with the measured values in Taurus and ChamI. This discrepancy, the absence of high-mass stars in USco-B, and its marginally distinct kinematics suggest that it might not be directly associated with either USco-A or Upper Centaurus Lupus, but instead might represent an older analog of the $\rho$ Oph or Lupus associations.

The authors thank R. White and C. Slesnick for helpful feedback on the manuscript and on various ideas presented within. 
This work makes use of data products from the Two Micron All Sky Survey, which is a joint project of the University of Massachusetts and the Infrared Processing and Analysis Center/ California Institute of Technology, funded by the National Aeronautics and Space Administration and the National Science
Foundation. This research has also made extensive use of the SIMBAD database, operated at CDS, Strasbourg, France, and of the USNOFS Image and Catalogue Archive, operated by the United States Naval Observatory, Flagstaff Station (http:// www.nofs.navy.mil/data/fchpix/).

\section{APPENDIX A}

\section{COMMENTS ON PROPER MOTIONS}

Photometric criteria are usually insufficient for identifying members of stellar populations. As we show in $\S 3$, near-infrared color cuts allow us to reject only $\sim 50 \%-90 \%$ of background and foreground contaminants. Any further increase in the rejection rate of our survey would require either spectroscopic observations (to test for lithium or signatures of low surface gravity, both indicators of youth) or astrometric observations (to measure proper motions and test for kinematic association). Performing spectroscopic observations can be a resource-intensive undertaking, but astrometric data are now commonly available from all-sky surveys.

The largest astrometric database currently available is the USNO-B catalog (Monet et al. 2003), which computed proper motions from the Palomar Observatory Sky Surveys of the mid-1950s and early 1980s. These observations were originally performed using wide-field photographic plates, and the USNO team digitally scanned these plates and computed photometry and astrometry for every source. The faint limit for photometry is $\sim R=19-20$, and astrometry is available for most sources brighter than $R=17-18$. Typical

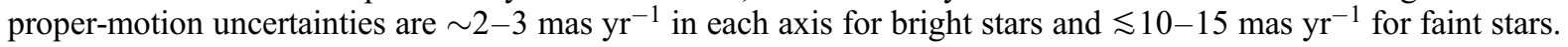

Since the USNO-B catalog is the result of an automated photometric/astrometric pipeline, individual measurements are subject to some uncertainties like distortions in the plate scale and centroid errors due to diffraction spikes from nearby bright stars. There are also some design issues that limit its utility. For example, objects with no proper-motion information (such as from detection in only one epoch)

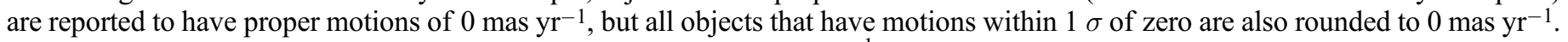
Thus, it is impossible to determine whether a measurement of 0 mas $\mathrm{yr}^{-1}$ corresponds to a bad measurement or a genuine detection of small proper motion. Finally, most stars within $<10^{\prime \prime}$ of a brighter object do not have proper-motion measurements available, so USNO-B astrometry is only potentially useful in studying wide companions.

In Figure 8 we present a plot of the fraction of confirmed Upper Sco members as a function of magnitude that possess any USNO-B

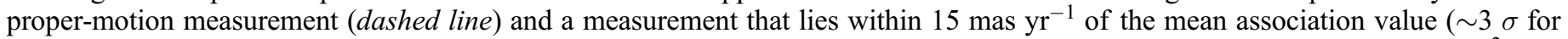
bright sources; solid line). The maximum fraction of confirmed members that are identified as kinematic members is only $\sim \frac{2}{3}$, and this fraction declines rapidly for faint targets $(K>12)$. This suggests that using existing proper motions to select candidate binary companions would introduce significant incompleteness in the resulting statistics, so we have chosen to omit these data from our selection criteria. However, these proper-motion measurements are useful as a test of our selection process, so we list the USNO-B proper motions for each candidate companion in Table 5. Those objects with consistent proper motions could be high-priority candidates for spectroscopic follow-up or more detailed astrometric follow-up. We also list the proper motions of each primary star in our sample in Table 2.

\section{APPENDIX B}

\section{THE KINEMATICS OF NORTHERN SCO-CEN}

The young stars of the Sco-Cen complex are divided into three subgroups: Upper Scorpius, Upper Centaurus Lupus, and Lower Centaurus Crux. These three subgroups are spatially distinct on the sky, but there is some overlap along the border between populations. This can lead to ambiguities in assigning stars to their appropriate population. For example, the stars of USco-B lie on the border between Upper Scorpius and Upper Centaurus Lupus. It is not known which group they are associated with, or if they form another distinct population. The color-magnitude sequences for each subgroup are not distinct due to their similar age and distance, so photometry does not provide a reliable diagnostic of subgroup membership. However, studies of the high-mass stars of Sco-Cen (e.g., de Zeeuw et al. 1999) have found that the space velocities of each subgroup differ by a few kilometers per second. This difference is not measurable in the proper motions of individual stars, but it might be detected as a difference in the mean proper motion for a population.

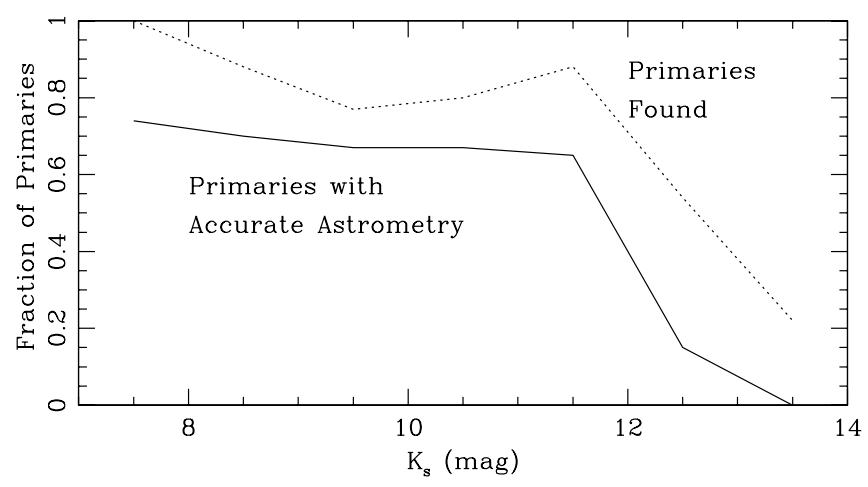

FIG. 8. - Plot of the fraction of confirmed Upper Sco members as a function of magnitude that possess proper-motion measurements in USNO-B (dashed line) and

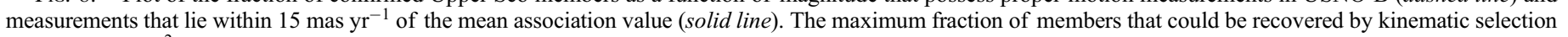
criteria is only $\sim \frac{2}{3}$, and this declines rapidly for faint targets $(K>12)$. 


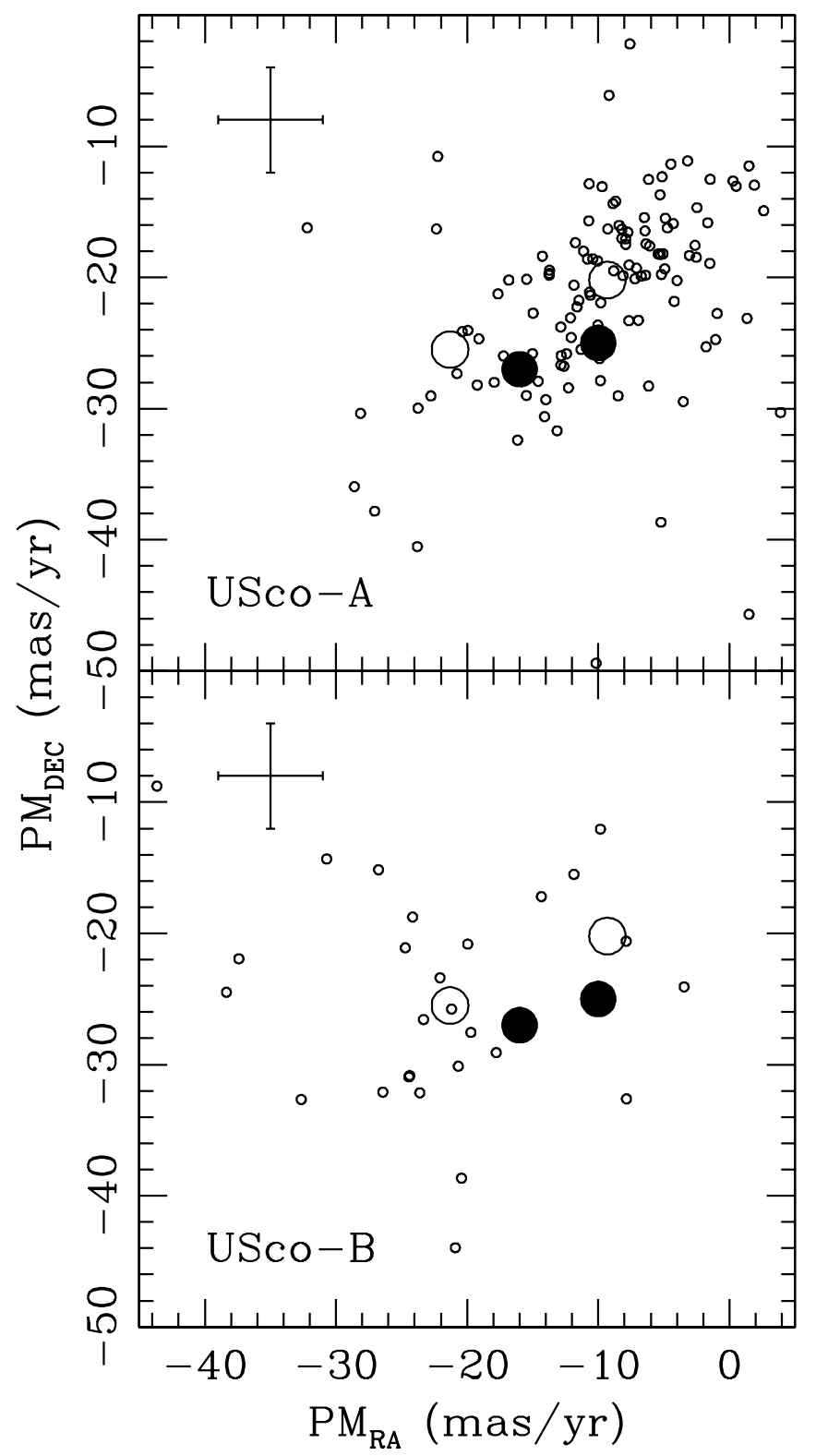

Fig. 9.- Proper-motion diagrams for Sco-Cen members brighter than $K=10$ that have been previously assigned to either USco-A or USco-B. The large filled circles denote the regional proper motions for Upper Sco $(-10,-25)$ and UCL $(-16,-27)$ as determined by Hipparcos for early-type members (de Zeeuw et al. 1999). The large open circles denote regional proper motions for USco-A $(-9.3,-20.2)$ and USco-B $(-21.3,-25.5)$ as determined from our data. The typical uncertainties for individual measurements are shown with error bars in the upper left corner; the scatter for USco-A appears to be consistent with these uncertainties, but the scatter for USco-B is

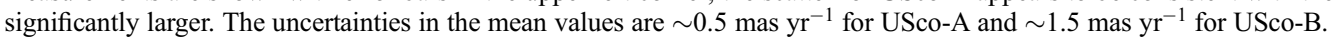

In Figure 9 we present proper-motion diagrams for USco members that have previously been assigned to USco-A or USco-B by Brandner et al. (1996) and Kohler et al. (2000). The mean proper motions for each subgroup are not directly comparable due to projection effects, but given the small radial velocity of USco-A $\left(-4.6 \mathrm{~km} \mathrm{~s}^{-1}\right.$; de Zeeuw et al. 1999) and the locations of the association centers $\left(16^{\mathrm{h}},-22^{\circ}\right.$ for USco-A; $15.5^{\mathrm{h}},-31^{\circ}$ for USco-B), the difference in proper motions should be no more than $2-3 \mathrm{mas}^{-1}$ and the vectors should be almost parallel. We find that the proper motion of USco-B $\left(33.2 \mathrm{mas} \mathrm{yr}^{-1}\right)$ is significantly higher than that of USco-A

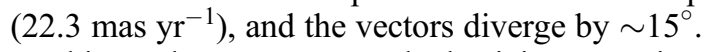

This result suggests not only that it is appropriate to consider USco-A and USco-B separately for statistical purposes, but that it might be prudent to question the relationship between USco-B and the rest of Sco-Cen. The difference in space velocities between USco-B and the other nearby Sco-Cen subgroups $\left(\sim 10 \mathrm{~km} \mathrm{~s}^{-1}\right)$ is far higher than that between the major subgroups. However, any further investigation is beyond the scope of this work.

We also conclude that the kinematic information lacks sufficient precision to distinguish the subgroup membership of individual stars and identify the boundary between the regions. Indeed, it is likely that there is no precise dividing line. The spatial distribution of these objects on the celestial sphere is only a projection of their three-dimensional distribution, so it is quite likely that the projected twodimensional distributions overlap. This suggests that any difference between these two populations could be averaged out by cross contamination. However, the distinct proper motions apparent in Figure 9 imply that most of the stars have been classified in the appropriate group. 


\section{APPENDIX C}

\section{THE NATURE OF UPPER SCORPIUS B}

The distinct binary properties observed for USco-A and USco-B suggest that it might be prudent to reconsider the nature of USco-B. The Sco-Cen complex consists primarily of three kinematically associated OB associations: Lower Centaurus Crux, Upper Centaurus Lupus, and Upper Scorpius. The $\rho$ Oph dark cloud complex is also associated with Sco-Cen (specifically with USco), and the Lupus dark clouds could be kinematically associated, but the evidence is not yet conclusive. LCC and UCL appear to be $\sim 5-10$ Myr older than USco, which in turn is $\sim 5$ Myr older than $\rho$ Oph. This has been cited as evidence (e.g., Mamajek et al. 2002; Sartori et al. 2003) that triggered star formation is occurring in Sco-Cen. The implication is that supernovae originating from the highest mass members of UCL triggered star formation in USco, and in turn one or more supernovae in USco triggered star formation in $\rho$ Oph. Since the ages of UCL and LCC are somewhat uncertain, it is unclear whether they are coeval or one triggered star formation in the other.

USco-B is located on the border between UCL and USco, in a region largely bereft of high-mass stars. Its age and distance are difficult to assess since there are no high-mass members that might possess Hipparcos distances, but the association's color-magnitude sequences lie slightly lower than USco-A and are consistent with the slightly larger distance and older age of UCL. However, its kinematics (Appendix A) appears to be marginally inconsistent with the OB subgroups of Sco-Cen, with a spatial velocity that differs by $\sim 10 \mathrm{~km} \mathrm{~s}^{-1}$. Finally, its binary properties are inconsistent with the one OB association that has been extensively studied (USco-A); no comparison is possible with UCL since there have been no large-scale surveys for new low-mass members.

The absence of high-mass stars and high wide binary frequency in USco-B is much more consistent with low-density Tassociations. This suggests that perhaps USco-B is an older analog to the $\rho$ Oph or Lupus clouds: an association consisting primarily of low-mass stars whose formation was triggered by supernovae in UCL, much as the current star formation in $\rho$ Oph was triggered by supernovae in USco, but which is not directly associated. Unfortunately, it will be difficult to test this assertion. Any primordial gas in USco-B has been dispersed, either consumed in star formation or swept away by supernovae and stellar winds, so it only consists of an unbound association of pre-main-sequence stars. The low galactic latitude of USco-B also results in significant contamination from background stars, which will confuse any photometric surveys that attempt to identify these stellar members of the association.

Abt, H., \& Levy, S. 1976, ApJS, 30, 273

Ardila, D., et al. 2000, AJ, 120, 479

Baraffe, I., Chabrier, G., Allard, F., \& Hauschildt, P. H. 1998, A\&A, 337, 403 2002, A\&A, 382, 563

Bate, M., \& Bonnell, I. 2005, MNRAS, 356, 1201

Baud, B., et al. 1984, ApJ, 278, L53

Bertiau, F. 1958, ApJ, 128, 533

Bertout, C., Robichon, N., \& Arenou, F. 1999, A\&A, 352, 574

Bessell, M., \& Brett, J. 1988, PASP, 100, 1134

Blaauw, A. 1946, Publ. Kapteyn Astron. Lab. Groningen, 52, 1

Bouy, H., et al. 2003, AJ, 126, 1526

2006a, A\&A, 451, 177

2006b, ApJ, 637, 1056

Brandner, W., et al. 1996, A\&A, 307, 121

Briceno, C., et al. 1993, PASP, 105, 686 1998, AJ, 115, 2074 2002, ApJ, 580, 317

Burgasser, A., et al. 2003, AJ, 125, 850

Cambresy, L., et al. 1998, A\&A, 338, 977

Carpenter, J., et al. 2002, AJ, 124, 1001

Chelli, A., et al. 1988, A\&A, 207, 46

Close, L., et al. 2003, ApJ, 587, 407 2005, Nature, 435, 287

Cohen, M., \& Kuhi, L. 1979, ApJS, 41, 743

Comerón, F., et al. 2004, A\&A, 417, 583

Cutri, R., et al. 2003, 2MASS All Sky Catalog of Point Sources

D’Antona, F., \& Mazzitelli, I. 1997, Mem. Soc. Astron. Italiana, 68, 807

de Geus, E., et al. 1989, A\&A, 216, 44

Delgado-Donate, E., et al. 2004, MNRAS, 347, 759

de Zeeuw, P., et al. 1999, AJ, 117, 354

Duchene, G. 1999, A\&A, 341, 547

Duquennoy, A., \& Mayor, M. 1991, A\&A, 248, 485

Fischer, D., \& Marcy, G. 1992, ApJ, 396, 178

Ghez, A., et al. 1993, AJ, 106, 2005 1997, ApJ, 481, 378

Glass, I. 1979, MNRAS, 187, 305

Goodwin, S., \& Kroupa, P. 2005, A\&A, 439, 565

Gregorio-Hetem, J., et al. 1992, AJ, 103, 549

Guieu, S., et al. 2006, A\&A, 446, 485

Haisch, K., et al. 2004, AJ, 127, 1747

Hartigan, P., et al. 1994, ApJ, 427, 961

Hartmann, L., et al. 2005, ApJ, 629, 881

Henize, K., \& Mendoza, E. 1973, ApJ, 180, 115

Herbig, G. 1952, J. R. Astron. Soc. Canada, 46, 222

Hillenbrand, L., \& White, R. 2004, ApJ, 604, 741

\section{REFERENCES}

Hubber, D., \& Whitworth, A. 2005, A\&A, 437, 113

Jones, B., \& Herbig, G. 1979, AJ, 84, 1872

Jones, D. 1971, MNRAS, 152, 231

Joy, A. 1945, ApJ, 102, 168

Kapteyn, J. 1914, ApJ, 40, 43

Kenyon, S., \& Hartmann, L. 1995, ApJS, 101, 117

Koenig, B., et al. 2001, A\&A, 369, 971

Kohler, R., et al. 2000, A\&A, 356, 541 2006, A\&A, 458, 461

Kouwenhoven, M., et al. 2005, A\&A, 430, 137

Kraus, A., et al. 2005, ApJ, 633, 452 2006, ApJ, 649, 306

Kroupa, P. 1995, MNRAS, 277, 1522

Kroupa, P., \& Bouvier, J. 2003, MNRAS, 346, 369

Kroupa, P., et al. 2003, MNRAS, 346, 354

Kunkel, M. 1999, Ph.D. thesis, Univ. Würzburg

Leggett, S., et al. 1998, ApJ, 509, 836

. 2000, ApJ, 535, 965

- 2001, ApJ, 548, 908

2002, ApJ, 564, 452

Leinert, Ch., et al. 1993, A\&A, 278, 129

Lopez-Marti, B., et al. 2004, A\&A, 416, 555

Luhman, K. 2000, ApJ, 544, 1044 2004a, ApJ, 602, 816 2004b, ApJ, 614, 398 2004c, ApJ, 617, 1216 2006, ApJ, 645, 676

Luhman, K., et al. 2003a, ApJ, 590, 348 2003b, ApJ, 593, 1093 2004, ApJ, 617, 565 2005, ApJ, 623, 1141

Mamajek, E., et al. 2002, AJ, 124, 1670

Martin, E., et al. 1998, MNRAS, 300, 733 2000, ApJ, 543, 299 2001, ApJ, 561, L195

\begin{tabular}{l} 
2003, ApJ, 594, 525 \\
\hline .2004, AJ, 127, 449
\end{tabular}

Masana, E., et al. 2006, A\&A, 450, 735

Metchev, S., et al. 2003, ApJ, 582, 1102

Mohanty, S., et al. 2005, ApJ, 626, 498

Monet, D., et al. 2003, AJ, 125, 984

Oasa, Y., et al. 1999, ApJ, 526, 336

Patience, J., et al. 2002, AJ, 123, 1570

Persi, P., et al. 2001, A\&A, 376, 907 - 2005, A\&A, 431, 539 
Preibisch, T., et al. 1998, A\&A, 333, 619 2001, AJ, 121, 1040 2002, AJ, 124, 404

Reid, I. N., \& Gizis, J. 1997, AJ, 113, 2246

Reid, I. N., \& Hawley, S. 1999, AJ, 117, 343

Reid, I. N., et al. 2001, AJ, 121, 489

Reiners, A., et al. 2005, ApJ, 634, 1346

Reipurth, B., \& Zinnecker, H. 1993, A\&A, 278, 81

Salim, S., \& Gould, A. 2003, ApJ, 582, 1011

Sartori, M., et al. 2003, A\&A, 404, 913

Schmidt-Kaler, Th. 1982, Physical Parameters of the Stars (Berlin: Springer)

Schwartz, R., et al. 1977, ApJS, 35, 161

Siegler, N., et al. 2005, ApJ, 621, 1023

Simon, M. 1997, ApJ, 482, L81

Simon, M., et al. 1995, ApJ, 443, 625

Skrutskie, M., et al. 2006, AJ, 131, 1163
Slesnick, C., et al. 2006a, AJ, 131, 3016 2006b, AJ, 132, 2665

Sterzik, M., \& Durisen, R. 1998, A\&A, 339, 95

Sterzik, M., et al. 2003, A\&A, 400, 1031

Stetson, P. 1987, PASP, 99, 191

Strom, K., \& Strom, S. 1994, ApJ, 424, 237

The, P.-S. 1964, PASP, 76, 293

Walter, F., et al. 1994, AJ, 107, 692

Weinberg, M., et al. 1987, ApJ, 312, 367

White, R., \& Basri, G. 2003, ApJ, 582, 1109

White, R., \& Ghez, A. 2001, ApJ, 556, 265

White, R., \& Hillenbrand, L. 2004, ApJ, 616, 998

Whittet, D., et al. 1997, A\&A, 327, 1194

Wichmann, R., et al. 1998, MNRAS, 301, L39

Woitas, J., et al. 2001, A\&A, 376, 982 